\title{
A PROFIT JUMP INVENTORY MODEL FOR IMPERFECT QUALITY ITEMS WITH RECEIVING REPARATIVE BATCH AND ORDER OVERLAPPING IN DENSE FUZZY ENVIRONMENT
}

\author{
Sujit Kumar De ${ }^{1}$ and Gour Chandra Mahata ${ }^{2, *}$
}

\begin{abstract}
This paper presents an economic order quantity (EOQ) inventory model for imperfect quality items with receiving a reparative batch and order overlapping in a dense fuzzy environment Here, the imperfect items are identified by screening and are divided into either scrap or reworkable items. The reworkable items are kept in store until the next items are received. Afterwards, the items are returned to the supplier to be reworked. Also, discount on the purchasing cost is employed as an offer of cooperation from a supplier to a buyer to compensate for all additional holding costs incurred to the buyer. The rework process is error free. An order overlapping scheme is employed so that the vendor is allowed to use the previous shipment to meet the demand by the inspection period. However, we assume the total monthly demand quantity as the dense fuzzy number because of learning effect. Moreover, first of all a profit maximization deterministic model is developed and solve by classical method. Fuzzifying the final optimized function via dense fuzzy demand quantity we have employed extended ranking index rule for its defuzzification. During the process of defuzzification we make an extensive study on the paradoxical unit square of the left and right deviations of dense fuzzy numbers. A comparative study is made after splitting the model into general fuzzy and dense fuzzy environment. Finally numerical and graphical illustrations and sensitivity analysis have been made for its global justifications.
\end{abstract}

Mathematics Subject Classification. 90B05.

Received April 9, 2019. Accepted February 9, 2021.

\section{INTRODUCTION}

The importance of inventory management systems is growing every day and many researchers are trying to solve management problems using mathematical models. The economic order quantity (EOQ) model is the basis of advanced inventory systems. By exploring the literature review on inventory systems, it is realized that many efforts have been conducted to provide inventory models in order to eliminate the limitations of the EOQ model. One of the assumptions in the EOQ models is that all the received items are perfect. However, this assumption is not comprehensive for several reasons that include faulty production process and failure in the process of transportation etc. So, the effect of imperfect items on inventory systems has become one of the interesting

Keywords. Inventory control, imperfect quality, order overlapping, dense fuzzy number, $(\rho, \sigma)-$ paradox.

1 Department of Mathematics, Midnapore College (Autonomous), Midnapore, Paschim Medinipur 721101, West Bengal, India.

2 Department of Mathematics, Sidho-Kanho-Birsha University, Sainik School, Ranchi Road, Purulia 723104, West Bengal, India.

*Corresponding author: gcmahata.skbu@gmail.com, gourmahata@yahoo.co.in 
topics for many researchers to provide more practical models. Porteus [40] followed by Rosenblatt and Lee [41] presented the significant connection between imperfect quality and lot sizing. Schwaller [44] assumed that the imperfect items received in the lot would result in the inspection cost. In addition, Zhang and Gerchak [56] studied an EOQ model with the effect of a joint lot sizing and screening, in which the imperfect items were random variables. Further, Salameh and Jaber [43] investigated an economic production quantity model for defective items with a known probability distribution. Therefore, they assumed that, by the end of the inspection time, the imperfect items were sold as a single batch. Cardenas-Barron did not deviate from the main idea, but pointed out and rectified an existing error in the model, which was devised by Salameh and Jaber [43]. Subsequently, Papachristos and Konstantaras [38] examined the imperfect inventory model given that the imperfect items were random variable. Moreover, Moussawi-Haidar et al. [36] suggested an inventory model in which lot-sizing, defective items, quality control were combined. Karimi-Nasab and Sabri-Laghaie [25] formulated a new imperfect production inventory model in which the imperfect items were randomly produced.

Recently, Moussawi-Haidar et al. [37] considered the effect of imperfect items and deterioration. It should be noted that, in all the above-mentioned models, no shortage has been assumed during the inspection process, which was based on the model by Salameh and Jaber [43]. Since in several successive inspection processes, defective items may have been found to cause shortage, the supposed assumption could not be correct. This fault was discussed by Papachristos and Konstantaras [38] who concluded that the simple formula could not be found to prevent the occurrence of shortage during the inspection process. Luckily, Maddah et al. [30] developed a pragmatic method to overcome this fault. This method, called "an order overlapping scheme", lets the vendor use the previous order to meet the demand during the inspection process. This new approach can effectively prevent the occurrence of shortage during the inspection process. Therefore, this idea was incorporated into our model.

Another unrealistic assumption considered in the above models was that imperfect goods could just be sold at their salvage value and could not be reworked. However, many researchers have discovered this fault and incorporated the idea of reworking a part of imperfect items into their models. For example, Hayek and Salameh [24] proposed an economic production quantity model, in which the defective items were reworked by the end of the production time. Yu et al. [53] studied an EOQ model in which a part of defective items could be used as good items. It should be noted that the above inventory models consider that the reworkable items are sent back to be reworked and returned as the perfect items through the same period; however in our model we have assumed that reworkable items were kept in the buyer's warehouse until the next shipment arrived. Then, the supplier replaced the reworkable items with the perfect ones and sent them within the next order before the current lot was used up. In the present paper, it was assumed that the following lot was received from the supplier as "reparative batch". Also, in the previous papers, it was assumed that the perfect item holding costs and scrap item holding costs were the same. Moreover, Wahab and Jaber [48] presented an imperfect EOQ inventory model with different holding costs and learning in the inspection. Again transporting items is a major concern so we may point the works of D'Ambrosio et al. [7] and Cerulli et al. [3] in this field of research also.

In all the above models, researchers have only considered all the parameters and variables as crisp values. Although crisp models offer an overview of the approach of inventory systems under various assumptions, they are not able to provide factual terms. As a result, exerting crisp models in general can lead to errors in decisionmaking. Also, in crisp models, inventory managers must be flexible in determining the economic lot size to cause non random uncertainty based cost reduction.

Zadeh [55] drew the concepts of fuzzy sets among researchers. Since then, Bellman and Zadeh [2] applied it in decision making for industrial management problems. In the inventory management topics Sommer [47] developed a fuzzy scheduling inventory model considering a constraint in warehouse capacity. Park [39] presented an EOQ model for interpreting a fuzzy set theory. Chang et al. [5] developed an EOQ inventory model considering the backorder as a triangular fuzzy number. Chang [4], Mahata and Goswami [33] proposed an imperfect inventory model considering the fuzzy annual demand and fuzzy imperfect rate. Mahata and Mahata [34] studied a fuzzy EOQ inventory model with two phase trade credits for deteriorating items in the fuzzy sense. 
In another study, Mahata [31] discussed an imperfect production model with partial backlogging of shortage quantity in fuzzy random environments. De and Sana [19] studied a hill type fuzzy stochastic model and got solutions via Bonferroni mean operator over score function of the fuzzy numbers. Furthermore, in the literature several ranking rules have been adopted by the researchers like Yager [52], Allahviranloo and Saneifard [1], Ezzati et al. [22], Deng [21] and Zhang et al. [57], etc. The concepts of deviation degree in fuzzy numbers were hosted by Wang et al. [49], Kumar et al. [29], Hajjari and Abbasbandy [23], Xu et al. [51], Yu et al. [54], etc. in developing the several inventory models. By this way numerous research articles along fuzzy environment have been studied ([8,11-20,32,35], etc.) yet. The main key factor of an inventory under smooth running is the supply of demand to the customers as quick as possible. But in reality we see due to lack of information the decision maker usually go for wrong decision in management system. So information gathering is one of the most essential part and parcel of any inventory process. In the literature, attempts have been taken by Kazemi et al. [26-28] to gain information through learning and forgetting process in fuzzy parameters for the backorder EOQ model with imperfect quality items. They applied Wright's [50] learning curve to gain knowledge in which the numbers of shipments are the vital factor. In another study, De and Mahata [11] discussed the learning effect on demand parameter in a backorder EOQ model through the duration of cycle time. Their basic notion is that, longer cycle time of an inventory can motivate customers in favour of that inventory spontaneously. They used the cloudy fuzzy approach on demand and compared the results with that of the general fuzzy model to justify their new approach. But in our study we have shown that customers/public interactions with the decision makers (DM) can change the motivation so that a catchment area over demand reaches very soon. In this study we have shown that adequate interaction could perform better goal in favour of inventory management system. However a reverse logic, more and more interactions of the DM to their learned customers can make a harmful situation (non favourable to DM) on the process itself. Also, such adequacy on interactions/negotiations/bargaining may vary from commodity to commodity, situations to situations even customer to customer or customer to DM implicitly. Thus we take the demand quantity as dense fuzzy number to estimate the actual learning outcomes in the inventory process itself. We have utilized De and Beg's [9, 10] ranking index rule to defuzzify the fuzzy objective function.

As it is obvious from the above-mentioned literature, none of the authors has presented an imperfect EOQ inventory model, either scrap or re-workable, along with receiving reparative batch considering various holding costs for perfect and scrap items under fuzzy conditions in the model parameters. Therefore, we tried to eliminate the gap in the literature. In this paper, scrap items were being sold for salvage value by the end of the inspection period. Upon the completion of the screening process, the buyer notifies the supplier the number of reworkable items; however, unlike some of the previous articles, here, it is assumed that reworkable items are stored in the buyer's warehouse until the next shipment arrives. Then, the supplier replaces the rework able items with the perfect ones and sends them within the next order before the current lot is exhausted. Totally, the major distinction between this paper and others lies in fuzziness in the demand parameter, the various assumptions on imperfect items, employing overlapping scheme to prevent shortages during the inspection period, discount rate provision of the purchasing cost to maintain a cooperative relationship, and considering receiving reparative. Moreover, we split the model into three different cases namely crisp, general fuzzy and dense fuzzy environments. Applying ranking index rule the new expressions for decision variables are developed. Numerical examples are also studied extensively. A comparative discussion along with sensitivity analysis and graphical illustrations are done to justify the new approach. At the end, a conclusion is made followed the scope of future work.

\section{Problem statement}

In this section, the problem is introduced with more details. An imperfect EOQ inventory model is presented. All the items received on a shipment are required to be inspected. The imperfect items that are identified through screening are divided into either scrap or rework able items. By the end of the inspection period, the scrap items are sold at a price of salvage value. Then, the buyer declares the number of rework able items; however unlike some of the previous articles in which rework able items are assumed to be sent back to the supplier and returned 
as the perfect items within the same period, the proposed model is assumed that rework able items are kept in a buyer's warehouse until the next shipment arrives. Then, the supplier replaces the rework able items with the perfect ones and sends them within the next order before the current lot is exhausted. By doing so, the supplier's costs (transportation costs) are reduced and, instead, the buyer's costs (holding costs) are raised. As a result, a coordinated policy should be employed so that economic benefits can be provided for both the buyer and the supplier. Discount on purchase costs can be used as an offer of cooperation from supplier to buyer (i.e., the discount compensates for all additional holding cost incurred to the buyer). Moreover, to eliminate shortages within the inspection period, an "overlapping scheme" is employed: similar to Maddah et al.'s [30] idea that let the buyer to supply his/her needs from the previous order during the inspection process. Also, it is assumed that the holding costs for scrap items and perfect items are not the same. The main objective of this article is to develop a profit function of an imperfect production process over the market of flexible demand designed by learning experiences of the decision maker. To do this we assume the demand parameter $D$ as triangular dense fuzzy number and to defuzzify the model we utilize the rule made by De and Beg [19].

Following are the assumptions and notations are considered in this paper:

\section{Assumptions}

- The input demand $D$ is the triangular dense fuzzy number.

- Shortages are not allowed.

- The holding cost for re-workable items is higher than that of scrap items.

- A discount on the purchasing cost is applied to meet up the extra holding cost belonging to the buyer.

- An order overlapping scheme is considered.

- The demand $(D)$ and screening processes $(x)$ proceed concurrently with $D<x$.

- Reworking is done instantly and the process is error-free.

\section{Notations}

The following notations are used to develop the model.

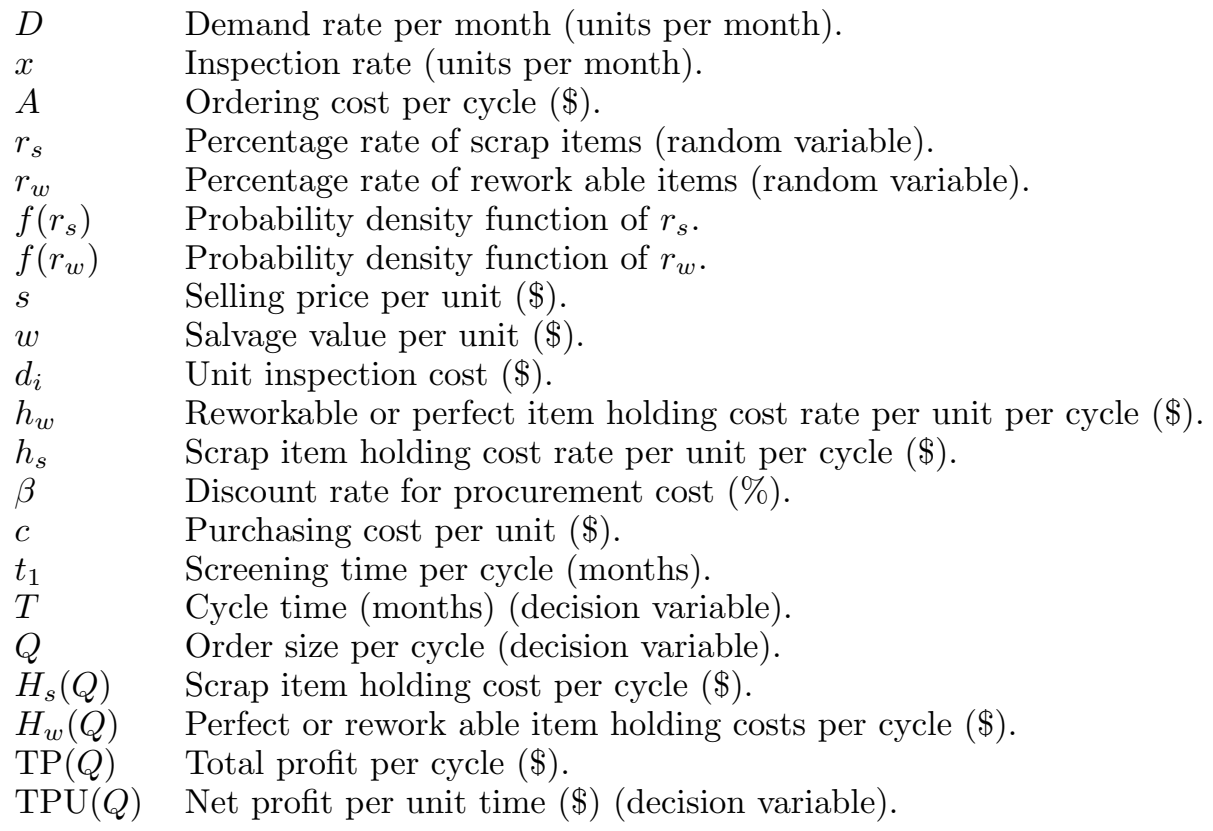




\section{Formulation of mathematical model (Extension of Maddah et al. [30])}

Considering above assumptions and notations we formulate the model and it is shown in Figure 1. We assume $100 \%$ inspection is finished at time $t_{1}$. To avoid shortages, the overlapping scheme is used and it is supposed that the demand by the screening time is at least the same as the number of perfect quality items. It means that, for $0 \leq t \leq t_{1}$ we have,

$$
x t_{1}\left(1-r_{s}-r_{w}\right) \geq D t_{1} \Rightarrow x \geq \frac{D}{1-r_{s}-r_{w}} .
$$

The goal is to obtain $Q$ that maximizes the total profit per year, $\operatorname{TP}(Q)$, expressed by

$$
\operatorname{TP}(Q)=\operatorname{TR}(Q)-\mathrm{TC}(Q)
$$

where $\operatorname{TR}(Q)$ denotes the revenue per cycle and $\mathrm{TC}(Q)$ denotes the total cost per cycle which is obtained through the sale of good items and scrap items. Thus, they can be defined respectively as follows:

$$
\operatorname{TR}(Q)=s Q\left(1-r_{s}\right)+w Q r_{s}
$$

and

$$
\mathrm{TC}(Q)=\mathrm{OC}+\mathrm{SC}+\mathrm{PC}+\mathrm{HC}
$$

where $\mathrm{OC}$ denotes the ordering cost per cycle $(\mathrm{OC}=A)$, SC denotes the screening cost per cycle $\left(\mathrm{SC}=d_{i} Q\right), \mathrm{PC}$ denotes the purchasing cost per cycle $(\mathrm{PC}=c Q(1-\beta))$, and $\mathrm{HC}$ denotes the holding cost per cycle, which includes the scrap item holding cost per cycle, $H_{s}(Q)$ and re-workable or perfect item holding cost per cycle, $H_{w}(Q) H_{s}(Q)$ can be obviously calculated using Figure 1 as shown in the shaded area:

$$
H_{s}(Q)=h_{s}\left(\frac{Q^{2} r_{s}}{2 x}\right)
$$

To compute $H_{w}(Q)$, the total inventory quantity per cycle should be calculated. According to Figure 1 , it is clear that the sum of the areas of $\triangle \mathrm{ZBC}, \triangle \mathrm{BGR}$, GIJR, and $\triangle \mathrm{RJF}$ minus $\triangle \mathrm{DEF}$ can express the total inventory quantity per cycle. The area of $\triangle \mathrm{ZBC}$ is the same as that of $\triangle \mathrm{DEF}$; therefore, we have:

$$
V=\frac{Q^{2} r_{s}}{\underbrace{2 x}_{\triangle \mathrm{BGR}}}+\frac{Q^{2}\left(1-r_{s}\right)}{\underbrace{x}_{\square \mathrm{GIJR}}}+\frac{Q^{2}\left(1-r_{s}\right)^{2}}{\underbrace{2 D}_{\triangle \mathrm{RJF}}} .
$$

Hence, the holding cost $H_{w}(Q)$ is given by

$$
H_{w}(Q)=h_{w} \times V=h_{w}\left[\frac{Q^{2} r_{s}}{2 x}+\frac{Q^{2}\left(1-r_{s}\right)}{x}+\frac{Q^{2}\left(1-r_{s}\right)}{2 D}\right] .
$$

Thus,

$$
\mathrm{TC}(Q)=A+d_{i} Q+c Q\left(1-\frac{r_{w} Q}{D}\right)+h_{s}\left(\frac{Q^{2} r_{s}}{2 x}\right)+h_{w}\left[\frac{Q^{2} r_{s}}{2 x}+\frac{Q^{2}\left(1-r_{s}\right)}{x}+\frac{Q^{2}\left(1-r_{s}\right)^{2}}{2 D}\right] .
$$

Through items simplification, the expression for total cost per cycle can be calculated by:

$$
\mathrm{TC}(Q)=A+d_{i} Q+c Q\left(1-\frac{r_{w} Q}{D}\right)+\left(h_{s}+h_{w}\right)\left(\frac{Q^{2} r_{s}}{2 x}\right)+h_{w}\left[\frac{Q^{2}\left(1-r_{s}\right)}{x}+\frac{Q^{2}\left(1-r_{s}\right)^{2}}{2 D}\right]
$$




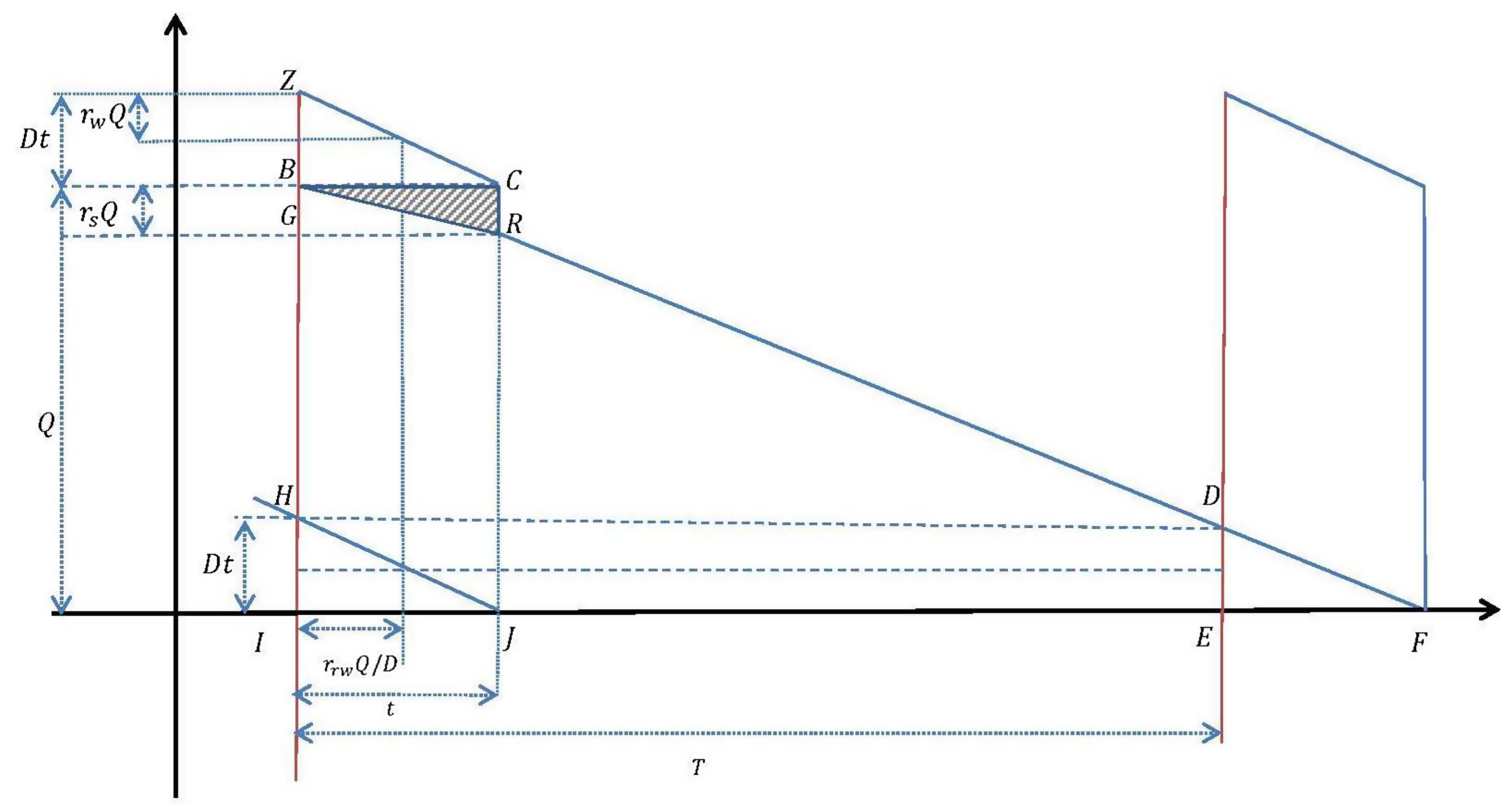

FiguRE 1. Imperfect EOQ model.

By substituting equations (3.8) and (3.3) into equation (3.2), the total profit per cycle is obtained by:

$$
\begin{aligned}
\operatorname{TP}(Q)= & s Q\left(1-r_{s}\right)+w Q r_{s}-A-d_{i} Q-c Q\left(1-\frac{r_{w} Q}{D}\right)-\left(h_{s}+h_{w}\right)\left(\frac{Q^{2} r_{s}}{2 x}\right) \\
& -h_{w}\left[\frac{Q^{2}\left(1-r_{s}\right)}{x}+\frac{Q^{2}\left(1-r_{s}\right)^{2}}{2 D}\right] .
\end{aligned}
$$

Furthermore, it is considered that the expected value of $\operatorname{TP}(Q)$, means $E[\operatorname{TP}(Q)]$ is calculated, in which the expected values $\left\{E\left(1-r_{s}\right), E\left(r_{s}\right)\right.$, and $\left.E\left(r_{w}\right)\right\}$ are used instead of $1-r_{s}, r_{s}$ and $r_{w}$, respectively. The expected net profit per unit time is calculated by applying the renewal reward theorem [42] (i.e., dividing TP $(Q)$ by the cycle length $T=\frac{\left(1-r_{s}\right) Q}{D}$ as follows:

$$
\begin{aligned}
E[\operatorname{TPU}(Q)]= & \frac{D\left[s\left\{1-E\left(r_{s}\right)\right\}+w E\left(r_{s}\right)-c\left\{\frac{1-E\left(r_{w}\right) Q}{D}\right\}-d_{i}\right]-\frac{A D}{Q}}{1-E\left(r_{s}\right)} \\
& -\frac{Q}{2\left(1-E\left(r_{s}\right)\right)}\left[\frac{D\left\{2 h_{w}-h_{w} E\left(r_{s}\right)+h_{s} E\left(r_{s}\right)\right\}}{x}+h_{w} E\left(1-r_{s}\right)^{2}\right] .
\end{aligned}
$$

Now, we consider:

$$
\left\{\begin{array}{l}
u=\frac{1}{1-E\left(r_{s}\right)}\left\{s\left\{1-E\left(r_{s}\right)\right\}+w E\left(r_{s}\right)-c-d_{i}-\frac{A}{Q}\right\} \\
W=\frac{Q}{2\left(1-E\left(r_{s}\right)\right)}\left\{\frac{2 h_{w}-h_{w} E\left(r_{s}\right)+h_{s} E\left(r_{s}\right)}{x}\right\} \\
X=\frac{h_{w} E\left(1-r_{s}\right)^{2}}{2\left(1-E\left(r_{s}\right)\right)}
\end{array} .\right.
$$

Hence, by substituting equation (3.12) in equation (3.11), the expected annual net profit function reduced to

$$
E[\operatorname{TPU}(Q)]=D u+\frac{c E\left(r_{w}\right)}{1-E\left(r_{s}\right)} Q-D W-Q X=D(u-W)+Q\left\{\frac{c E\left(r_{w}\right)}{1-E\left(r_{s}\right)}-X\right\} .
$$


Now to have the optimum value of the order quantity, we differentiate (3.13) with respect to $Q$ and get the following:

$$
\frac{\mathrm{d}\{E[\mathrm{TPU}(Q)]\}}{\mathrm{d} Q}=D\left(\frac{\mathrm{d} u}{\mathrm{~d} Q}-\frac{\mathrm{d} W}{\mathrm{~d} Q}\right)+\frac{c E\left(r_{w}\right)}{1-E\left(r_{s}\right)}-X=0
$$

where,

$$
\frac{\mathrm{d} u}{\mathrm{~d} Q}=\frac{\mathrm{d}}{\mathrm{d} Q}\left\{\frac{1}{1-E\left(r_{s}\right)}\left\{s\left\{1-E\left(r_{s}\right)\right\}+w E\left(r_{s}\right)-c-d_{i}-\frac{A}{Q}\right\}\right\}=\frac{A}{Q\left\{1-E\left(r_{s}\right)\right\}}
$$

and

$$
\frac{\mathrm{d} W}{\mathrm{~d} Q}=\frac{\mathrm{d}}{\mathrm{d} Q}\left\{\frac{Q}{2\left(1-E\left(r_{s}\right)\right)}\left\{\frac{2 h_{w}-h_{w} E\left(r_{s}\right)+h_{s} E\left(r_{s}\right)}{x}\right\}\right\}=\left\{\frac{2 h_{w}-h_{w} E\left(r_{s}\right)+h_{s} E\left(r_{s}\right)}{2 x\left(1-E\left(r_{s}\right)\right)}\right\} .
$$

Now, substituting (3.15) and (3.16) in (3.14), we have,

$$
D\left[\frac{A}{Q^{2}\left\{1-E\left(r_{s}\right)\right\}}-\frac{2 h_{w}-h_{w} E\left(r_{s}\right)+h_{s} E\left(r_{s}\right)}{2 x\left(1-E\left(r_{s}\right)\right)}\right]+\frac{c E\left(r_{w}\right)}{1-E\left(r_{s}\right)}-\frac{h_{w} E\left(1-r_{s}\right)^{2}}{2\left(1-E\left(r_{s}\right)\right)}=0 .
$$

Simplifying (3.17) we get $\frac{A}{Q^{2}}=-\frac{c E\left(r_{w}\right)}{D}+\frac{h_{w} E\left(1-r_{s}\right)^{2}}{2 D}+\frac{2 h_{w}-h_{w} E\left(r_{s}\right)+h_{s} E\left(r_{s}\right)}{2 x}$ giving

$$
Q^{*}=\sqrt{\frac{2 A D}{D\left\{\frac{2 h_{w}-h_{w} E\left(r_{s}\right)+h_{s} E\left(r_{s}\right)}{2 x}\right\}-2 c E\left(r_{w}\right)+h_{w} E\left(1-r_{s}\right)^{2}}}
$$

and this value yields

$$
\frac{\mathrm{d}^{2}\{E[\operatorname{TPU}(Q)]\}}{\mathrm{d} Q^{2}}=\frac{-2 A D}{Q^{2}\left\{1-E\left(r_{s}\right)\right\}}<0 .
$$

This confirms that the objective function has global maximum and its value is given by

$$
E[\operatorname{TPU}(Q)]^{*}=D\left[F_{1}-\frac{F_{2}}{Q^{*}}-F_{3} Q^{*}\right]-F_{4} Q^{*}
$$

where

$$
\left\{\begin{array}{l}
F_{1}=\frac{s\left\{1-E\left(r_{s}\right)\right\}+w E\left(r_{s}\right)-c-d_{i}}{1-E\left(r_{s}\right)} \\
F_{2}=\frac{A}{1-E\left(r_{s}\right)} \\
F_{3}=\frac{2 h_{w}-h_{w} E\left(r_{s}\right)+h_{s} E\left(r_{s}\right)}{2\left\{1-E\left(r_{s}\right)\right\} x} \\
F_{4}=\frac{h_{w} E\left(1-r_{s}\right)^{2}}{2\left\{1-E\left(r_{s}\right)\right\}}-\frac{c E\left(r_{w}\right)}{\left\{1-E\left(r_{s}\right)\right\}}
\end{array} .\right.
$$

Note that, when the demand parameter $D$ is finite and screening rate is large enough, that is the inspection process is finished simultaneously by the receiving an order, and finally when items are categorized as only perfect or imperfect (no rework able items so that no discount on purchasing cost), then $Q^{*}$ in equation (3.18) is equivalent to

$$
Q^{*}=\sqrt{\frac{2 A D}{h_{w} E\left(1-r_{s}\right)^{2}}}
$$

This is the same as the results obtained by Shih [45] and Silver [46]. It shows that the proposed model is accurate. In addition, it should be noted that, when the demand parameter $D$ is finite, and if all items are assumed to be perfect, our model becomes an equivalent to the EOQ inventory model. 


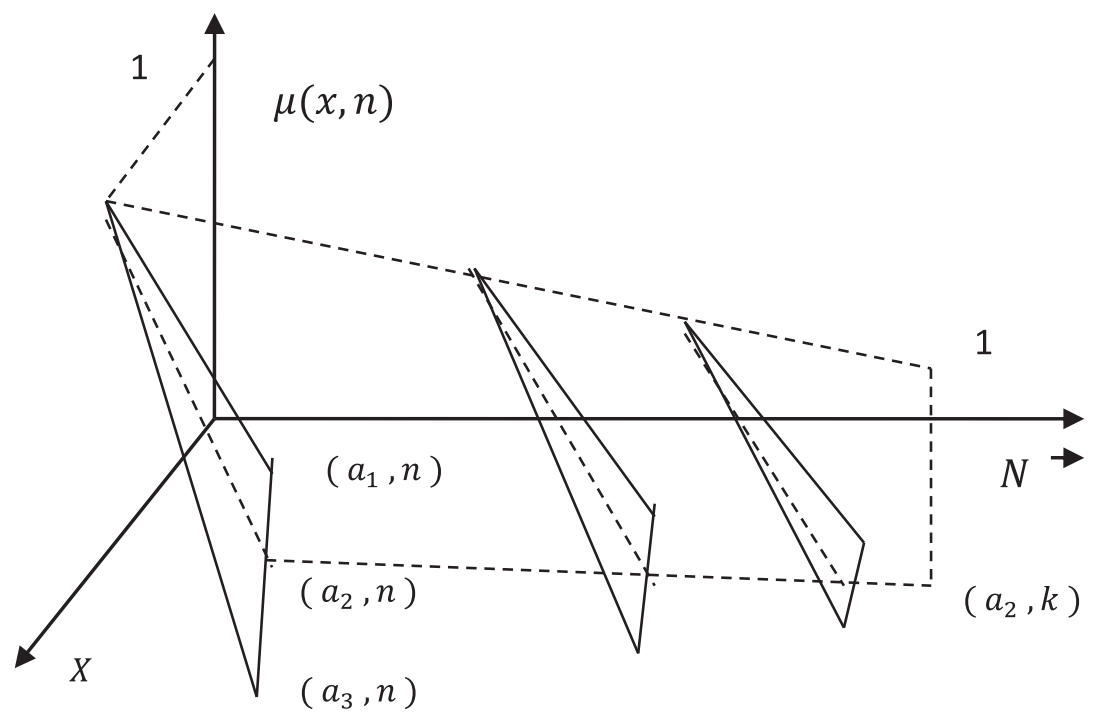

Figure 2. Membership function of TDFN.

\section{FuZzy MATHEMATiCAL MODEL}

Let the demand rate assumes flexible under dense fuzzy environment. Then the fuzzy problem (the tilt $\mathrm{bar}^{\sim}$ represents the fuzzification of the parameters) corresponding to the crisp model (3.20) is given by

$$
\begin{aligned}
& \text { Maximize } \widetilde{Z_{E}} \cong \widetilde{D}\left[F_{1}-\frac{F_{2}}{\widetilde{Q^{*}}}-F_{3} \widetilde{Q^{*}}\right]-F_{4} \widetilde{Q^{*}} \\
& \text { Subject to }\left\{\begin{array}{l}
\widetilde{Q^{*}}=\sqrt{\frac{2 A \widetilde{D}}{\widetilde{D} Y_{1}+Y_{2}}} \\
\widetilde{T^{*}}=\frac{\left(1-r_{s}\right) \widetilde{Q^{*}}}{\widetilde{D}}
\end{array}\right. \\
& \text { where }\left\{\begin{array}{l}
Y_{1}=\frac{2 h_{w}-h_{w} E\left(r_{s}\right)+h_{s} E\left(r_{s}\right)}{x} \\
Y_{2}=h_{w} E\left(1-r_{s}\right)^{2}-2 c E\left(r_{w}\right)
\end{array}\right.
\end{aligned}
$$

and the other parameters are obtained from (3.21).

Now, as per De and Beg $[9,10]$ we use the following membership function for dense fuzzy demand rate as

$$
\mu(D, n)= \begin{cases}0 & \text { if } D<D_{2}\left(1-\frac{\rho}{1+n}\right) \text { and } D>D_{2}\left(1-\frac{\sigma}{1+n}\right) \\ \left\{\frac{D-D_{2}\left(1-\frac{\rho}{1+n}\right)}{\left.\frac{\rho D_{2}}{1+n}\right\}}\right. & \text { if } D_{2}\left(1-\frac{\rho}{1+n}\right) \leq D \leq D_{2} \\ \left\{\frac{D_{2}\left(1+\frac{\sigma}{1+n}\right)-D}{\left.\frac{\sigma D_{2}}{1+n}\right\}}\right. & \text { if } D_{2} \leq D \leq D_{2}\left(1+\frac{\sigma}{1+n}\right)\end{cases}
$$

where, $0<\rho, \sigma<1$ and $n$ being the natural number and the graphical representation of this triangular dense fuzzy number (TDFN) is given in Figure 2. 
Therefore, to get the membership value of the optimum order quantity $Q^{*}$ we need to proceed as follows: From (4.4), we write for all positive numbers as follows:

$$
D_{2}\left(1-\frac{\rho}{1+n}\right) \leq D \leq D_{2} \Rightarrow \sqrt{\frac{2 A D_{2}\left(1-\frac{\rho}{1+n}\right)}{D_{2} Y_{1}+Y_{2}}} \leq \sqrt{\frac{2 A D}{D Y_{1}+Y_{2}}} \leq \sqrt{\frac{2 A D_{2}}{D_{2} Y_{1}\left(1-\frac{\rho}{1+n}\right)+Y_{2}}}
$$

and

$$
D_{2} \leq D \leq D_{2}\left(1+\frac{\sigma}{1+n}\right) \Rightarrow \sqrt{\frac{2 A D_{2}}{D_{2} Y_{1}\left(1+\frac{\sigma}{1+n}\right)+Y_{2}}} \leq \sqrt{\frac{2 A D}{D Y_{1}+Y_{2}}} \leq \sqrt{\frac{2 A D_{2}\left(1+\frac{\sigma}{1+n}\right)}{D_{2} Y_{1}+Y_{2}}}
$$

Thus,

$$
\begin{gathered}
\sqrt{\frac{2 A D_{2}\left(1-\frac{\rho}{1+n}\right)}{D Y_{1}+Y_{2}}} \leq Q^{*} \leq \sqrt{\frac{2 A D_{2}}{D_{2} Y_{1}\left(1-\frac{\rho}{1+n}\right)+Y_{2}}} \text { and } \\
\sqrt{\frac{2 A D_{2}}{D_{2} Y_{1}\left(1-\frac{\rho}{1+n}\right)+Y_{2}}} \leq Q^{*} \leq \sqrt{\frac{2 A D_{2}\left(1+\frac{\sigma}{1+n}\right)}{D Y_{1}+Y_{2}}}
\end{gathered}
$$

because $\sqrt{\frac{2 A D_{2}}{D_{2} Y_{1}\left(1-\frac{\rho}{1+n}\right)+Y_{2}}}>\sqrt{\frac{2 A D_{2}}{D_{2} Y_{1}\left(1+\frac{\sigma}{1+n}\right)+Y_{2}}}$.

Let us assume $Q_{1} \leq Q^{*} \leq Q_{2}$ and $Q_{2} \leq Q^{*} \leq Q_{3}$.

So

$$
\left\{\begin{array}{l}
Q_{1}=\sqrt{\frac{2 A D_{2}\left(1-\frac{\rho}{1+n}\right)}{D Y_{1}+Y_{2}}} \\
Q_{2}=\sqrt{\frac{2 A D_{2}}{D_{2} Y_{1}\left(1+\frac{\sigma}{1+n}\right)+Y_{2}}} . \\
Q_{3}=\sqrt{\frac{2 A D_{2}\left(1+\frac{\sigma}{1+n}\right)}{D Y_{1}+Y_{2}}}
\end{array}\right.
$$

And the corresponding membership function of the optimum order quantity is given by

$$
\mu\left(Q^{*}, n\right)=\left\{\begin{array}{ll}
0 & \text { if } Q^{*}<Q_{2} \text { and } Q^{*}>Q_{3} \\
\frac{Q^{*}-Q_{1}}{Q_{2}-Q_{1}} & \text { if } Q_{1} \leq Q^{*} \leq Q_{2} \\
\frac{Q_{3}-Q^{*}}{Q_{3}-Q_{2}} & \text { if } Q_{2} \leq Q^{*} \leq Q_{3}
\end{array} .\right.
$$

Now, applying the fuzzy arithmetic (for details see Appendix A.1), the net membership of the optimal objective we get,

$$
\begin{aligned}
\mu\left(\widetilde{Z_{E}}\right) & =\mu\left(F_{1} \widetilde{D}\right)-\mu\left(F_{2} \widetilde{D} / \widetilde{Q^{*}}\right)-\mu\left(F_{3} \widetilde{D} \widetilde{Q^{*}}\right)-\mu\left(F_{4} \widetilde{Q^{*}}\right) \\
\text { and } \mu\left(\widetilde{T^{*}}\right) & =\mu\left[\frac{\left(1-r_{s}\right) \widetilde{Q^{*}}}{\widetilde{D}}\right] .
\end{aligned}
$$

Now, the $\alpha$-cuts of $\mu\left(\widetilde{Z_{E}}\right)$ and $\mu\left(\widetilde{T^{*}}\right)$ are respectively given by,

$$
\left[Z_{\mathrm{EL}}^{*}, Z_{\mathrm{ER}}^{*}\right]=F_{1}\left[D_{1}+\alpha\left(D_{2}-D_{1}\right), D_{3}-\alpha\left(D_{3}-D_{2}\right)\right]-F_{2}\left[\frac{D_{1}+\alpha\left(D_{2}-D_{1}\right)}{Q_{3}-\alpha\left(Q_{3}-Q_{2}\right)}, \frac{D_{3}-\alpha\left(D_{3}-D_{2}\right)}{Q_{1}+\alpha\left(Q_{2}-Q_{1}\right)}\right]
$$




$$
\begin{aligned}
& -F_{3}\left[\left\{D_{1}+\alpha\left(D_{2}-D_{1}\right)\right\}\left\{Q_{1}+\alpha\left(Q_{2}-Q_{1}\right)\right\},\left\{D_{3}-\alpha\left(D_{3}-D_{2}\right)\right\}\left\{Q_{3}-\alpha\left(Q_{3}-Q_{2}\right)\right\}\right] \\
& -F_{4}\left[Q_{1}+\alpha\left(Q_{2}-Q_{1}\right), Q_{3}-\alpha\left(Q_{3}-Q_{2}\right)\right] \\
\text { and } \quad\left[T_{L}^{*}, T_{R}^{*}\right]= & \left(1-r_{s}\right)\left[\frac{Q_{1}+\alpha\left(Q_{2}-Q_{1}\right)}{D_{3}-\alpha\left(D_{3}-D_{2}\right)}, \frac{Q_{3}-\alpha\left(Q_{3}-Q_{2}\right)}{D_{1}+\alpha\left(D_{2}-D_{1}\right)}\right]
\end{aligned}
$$

Therefore, after little calculations, (4.9) and (4.10) reduces to

$$
\begin{aligned}
I\left(Z_{E}^{*}\right)= & \frac{1}{2 K} \sum_{n=0}^{K} \int_{0}^{1}\left[Z_{\mathrm{EL}}^{*}+Z_{\mathrm{ER}}^{*}\right] \mathrm{d} \alpha=\frac{F_{1}}{4 K} \sum_{n=0}^{K}\left(D_{1}+2 D_{2}+D_{3}\right)-\frac{F_{4}}{4 K} \sum_{n=0}^{K}\left(Q_{1}+2 Q_{2}+Q_{3}\right) \\
& -\frac{F_{2}}{2 K} \sum_{n=0}^{K} \int_{0}^{1}\left[\frac{D_{1}+\alpha\left(D_{2}-D_{1}\right)}{Q_{3}-\alpha\left(Q_{3}-Q_{2}\right)}+\frac{D_{3}-\alpha\left(D_{3}-D_{2}\right)}{Q_{1}+\alpha\left(Q_{2}-Q_{1}\right)}\right] \mathrm{d} \alpha \\
& -\frac{F_{3}}{12 K} \sum_{n=0}^{K}\left[Q_{3} D_{2}+Q_{2} D_{3}+2 Q_{3} D_{3}+4 Q_{2} D_{2}+2 Q_{1} D_{1}+Q_{1} D_{2}+Q_{2} D_{1}\right] \\
= & \frac{F_{1}}{4 K} \sum_{n=0}^{K}\left(D_{1}+2 D_{2}+D_{3}\right)-\frac{F_{4}}{4 K} \sum_{n=0}^{K}\left(Q_{1}+2 Q_{2}+Q_{3}\right) \\
& -\frac{F_{3}}{12 K} \sum_{n=0}^{K}\left[Q_{3} D_{2}+Q_{2} D_{3}+2 Q_{3} D_{3}+4 Q_{2} D_{2}+2 Q_{1} D_{1}+Q_{1} D_{2}+Q_{2} D_{1}\right] \\
& -\frac{F_{2}}{2 K} \sum_{n=0}^{K}\left[\frac{Q_{2} D_{3}-Q_{1} D_{2}}{\left(Q_{2}-Q_{1}\right)^{2}} \log \left(\frac{Q_{2}}{Q_{1}}\right)-\frac{Q_{3} D_{2}-Q_{2} D_{1}}{\left(Q_{3}-Q_{2}\right)^{2}} \log \left(\frac{Q_{2}}{Q_{3}}\right)\right. \\
& -\frac{Q_{3} D_{3}+2 Q_{2} D_{2}+Q_{1} D_{1}-Q_{1} D_{2}-Q_{2} D_{1}-Q_{3} D_{2}-Q_{2} D_{3}}{Q_{1} Q_{2}+Q_{3} Q_{2}-Q_{3} Q_{1}-Q_{2}^{2}} .
\end{aligned}
$$

And that for order quantity is given by

$$
I\left(Q^{*}\right)=\frac{1}{4 K} \sum_{n=0}^{K}\left(Q_{1}+2 Q_{2}+Q_{3}\right)
$$

and

$$
\begin{aligned}
I\left(T^{*}\right)= & \frac{\left(1-r_{s}\right)}{2 K} \sum_{n=0}^{K}\left[\frac{D_{2} Q_{3}-D_{1} Q_{2}}{\left(D_{2}-D_{1}\right)^{2}} \log \left(\frac{D_{2}}{D_{1}}\right)-\frac{D_{3} Q_{2}-D_{2} Q_{1}}{\left(D_{3}-D_{2}\right)^{2}} \log \left(\frac{D_{2}}{D_{3}}\right)\right. \\
& \left.-\frac{D_{3} Q_{3}+2 Q_{2} D_{2}+Q_{1} D_{1}-D_{1} Q_{2}-D_{2} Q_{1}-D_{3} Q_{2}-Q_{2} D_{3}}{D_{1} D_{2}+D_{3} D_{2}-D_{3} D_{1}-D_{2}^{2}}\right] .
\end{aligned}
$$

However,

$$
D_{1}+2 D_{2}+D_{3}=D_{2}\left\{4+\frac{\sigma-\rho}{1+n}\right\}
$$

and

$$
Q_{1}+2 Q_{2}+Q_{3}=\sqrt{\frac{2 A D_{2}\left(1-\frac{\rho}{1+n}\right)}{D_{2} Y_{1}+Y_{2}}}+2 \sqrt{\frac{2 A D_{2}}{D_{2} Y_{1}\left(1+\frac{\sigma}{1+n}\right)+Y_{2}}}+\sqrt{\frac{2 A D_{2}\left(1+\frac{\sigma}{1+n}\right)}{D_{2} Y_{1}+Y_{2}}}
$$




$$
=\lambda\left[\sqrt{1+\frac{\sigma}{1+n}}+\sqrt{1-\frac{\rho}{1+n}}+2 \sqrt{\frac{1}{1+\frac{\sigma}{1+n} \frac{Y_{1} \lambda^{2}}{2 A}}}\right] .
$$

Letting, $\lambda=\sqrt{\frac{2 A D_{2}}{D_{2} Y_{1}+Y_{2}}}$ we have

$I\left(Z_{E}^{*}\right)$

$$
\begin{aligned}
& =\frac{F_{1} d_{2}}{4 K} \sum_{n=0}^{K}\left\{4+\frac{\sigma-\rho}{1+n}\right\}-\frac{F_{4} \lambda}{4 K} \sum_{n=0}^{K}\left[\sqrt{1+\frac{\sigma}{1+n}}+\sqrt{1-\frac{\rho}{1+n}}+2 \sqrt{\frac{1}{1+\frac{\sigma}{1+n} \frac{Y_{1} \lambda^{2}}{2 A}}}\right] \\
& -\frac{d_{2} F_{2}}{4 \lambda K} \sum_{n=0}^{K}\left[\begin{array}{l}
+\sqrt{\left(1+\frac{\sigma}{1+n} \frac{Y_{1} \lambda^{2}}{2 A}\right)}\left\{\frac{\left(1+\frac{\sigma}{1+n}-\sqrt{\left(1-\frac{\rho}{1+n}\right)\left(1+\frac{\sigma}{1+n} \frac{Y_{1} \lambda^{2}}{2 A}\right)}\right)}{\left.\left(1-\sqrt{\left(1-\frac{\rho}{1+n}\right)\left(1+\frac{\sigma}{1+n} \frac{Y_{1} \lambda^{2}}{2 A}\right.}\right)\right)^{2}}\right\} \log \left|\left(1+\frac{\sigma}{1+n} \frac{Y_{1} \lambda^{2}}{2 A}\right)\left(1-\frac{\rho}{1+n}\right)\right| \\
+\sqrt{\left(1+\frac{\sigma}{1+n} \frac{Y_{1} \lambda^{2}}{2 A}\right)}\left\{\frac{\left(\sqrt{\left(1+\frac{\sigma}{1+n}\right)\left(1+\frac{\sigma}{1+n} \frac{Y_{1} \lambda^{2}}{2 A}\right)}-1+\frac{\rho}{1+n}\right)}{\left(\sqrt{\left(1+\frac{\sigma}{1+n}\right)\left(1+\frac{\sigma}{1+n} \frac{Y_{1} \lambda^{2}}{2 A}\right)}-1\right)^{2}}\right\} \log \left|\left(1+\frac{\sigma}{1+n} \frac{Y_{1} \lambda^{2}}{2 A}\right)\left(1+\frac{\sigma}{1+n}\right)\right| \\
-2\left\{\frac{\frac{\rho-\sigma}{1+n} \sqrt{\left(1+\frac{\sigma}{1+n} \frac{Y_{1} \lambda^{2}}{2 A}\right)}+\left(1+\frac{\sigma}{1+n} \frac{Y_{1} \lambda^{2}}{2 A}\right)\left\{\frac{\sigma}{1+n} \sqrt{1+\frac{\sigma}{1+n}}-\frac{\rho}{1+n} \sqrt{\left(1-\frac{\rho}{1+n}\right)}\right\}}{\sqrt{\left(1+\frac{\sigma}{1+n} \frac{Y_{1} \lambda^{2}}{2 A}\right)}\left\{\sqrt{1+\frac{\sigma}{1+n}}+\sqrt{\left(1-\frac{\rho}{1+n}\right)}\right\}-1-\left(1+\frac{\sigma}{1+n} \frac{Y_{1} \lambda^{2}}{2 A}\right) \sqrt{\left(1+\frac{\sigma}{1+n}\right)\left(1-\frac{\rho}{1+n}\right)}}\right\}
\end{array}\right] \\
& -\frac{\lambda d_{2} F_{3}}{12 K} \sum_{n=0}^{K}\left[\sqrt{1+\frac{\sigma}{1+n}}+\frac{\left(6+\frac{\sigma-\rho}{1+n}\right)}{\sqrt{\left(1+\frac{\sigma}{1+n} \frac{Y_{1} \lambda^{2}}{2 A}\right)}}+2\left(1+\frac{\sigma}{1+n}\right)^{3 / 2}+2\left(1-\frac{\rho}{1+n}\right)^{3 / 2}+\sqrt{1-\frac{\rho}{1+n}}\right]
\end{aligned}
$$

and

$$
\begin{aligned}
& I\left(Q^{*}\right)=\frac{1}{4 K} \sum_{n=0}^{K}\left(Q_{1}+2 Q_{2}+Q_{3}\right)=\frac{\lambda}{4 K} \sum_{n=0}^{K}\left[\sqrt{1+\frac{\sigma}{1+n}}+\sqrt{1-\frac{\rho}{1+n}}+2 \sqrt{\frac{1}{1+\frac{\sigma}{1+n} \frac{Y_{1} \lambda^{2}}{2 A}}}\right] \\
& I\left(T^{*}\right)=\frac{\left(1-r_{s}\right) \lambda}{2 K D} \sum_{n=0}^{K}\left[\frac{\sqrt{\left(1+\frac{\sigma}{1+n}\right)}-\left(1+\frac{\rho}{1+n}\right) \sqrt{\frac{1}{1+\frac{\sigma}{1+n} \frac{Y_{1} \lambda^{2}}{2 A}}}}{\left(\frac{\rho}{1+n}\right)^{2}} \log \left|\frac{1}{1-\frac{\rho}{1+n}}\right|\right. \\
& +\frac{\left(1+\frac{\sigma}{1+n}\right) \sqrt{\frac{1}{1+\frac{\sigma}{1+n} \frac{Y_{1} \lambda^{2}}{2 A}}}-\sqrt{\left(1+\frac{\rho}{1+n}\right)}}{\left(\frac{\sigma}{1+n}\right)^{2}} \log \left|1+\frac{\sigma}{1+n}\right| \\
& \left.-\frac{\left(\frac{\sigma}{1+n}\right) \sqrt{\left(1+\frac{\sigma}{1+n}\right)}-\frac{\rho}{1+n} \sqrt{\left(1+\frac{\rho}{1+n}\right)}+\left(\frac{\rho-\sigma}{1+n}\right) \sqrt{\frac{1}{1+\frac{\sigma}{1+n} \frac{Y_{1} \lambda^{2}}{2 A}}}}{\frac{\sigma \rho}{(1+n)^{2}}}\right] .
\end{aligned}
$$

Now we shall solve the above objective function (4.16) subject to the condition (4.17) and (4.18) in three different cases:

Case I: Crisp model.

Case II: General fuzzy model for $\rho>\sigma, \rho=\sigma$ and $\rho<\sigma$ (putting $n=0$ in dense fuzzy model).

Case III: Dense fuzzy model for $\rho>\sigma, \rho=\sigma$ and $\rho<\sigma$. 
TABLE 1. Optimal solution for general fuzzy model.

\begin{tabular}{lllll}
\hline \hline Left and right deviations & $I\left(Q^{*}\right)$ & $I\left(T^{*}\right)$ & $I\left(Z_{E}^{*}\right)$ & $R_{z}=\left\{\frac{I\left(Z_{E}^{*}\right)-Z_{E}^{*}}{Z_{E}^{*}}\right\} \times 100 \%$ \\
\hline$\rho(=.2)<\sigma(=.3)$ & 870.001 & 10.288 & 23869.50 & 2.46 \\
$\rho=\sigma=.2$ & 873.971 & 10.494 & 23289.22 & -0.03 \\
$\rho(=.3)>\sigma(=.2)$ & 860.835 & 10.767 & 22700.48 & -2.56 \\
\hline
\end{tabular}

TABLE 2. Optimal solution for dense fuzzy model.

\begin{tabular}{lllll}
\hline \hline Left and right deviations & $n$ & $I\left(Q^{*}\right)$ & $I\left(T^{*}\right)$ & $I\left(Z_{E}^{*}\right)$ \\
\hline$\rho<\sigma$ & & 1758.71 & 20.69 & 1417.27 \\
$\rho=\sigma$ & 1 & 1765.401 & 21.0 & 43408.94 \\
$\rho>\sigma$ & & 1746.186 & 21.4 & 44156.25 \\
\hline
\end{tabular}

\section{NumericAl EXAMPLE 1}

Suppose the inventory practitioner (DM) ordered a single item which is purchased and sold batch wise. These are combination of scrap rework able and good items. The monthly demand of this item is found to be 1000 units $(=D)$ and the inspection rate is 10000 units $(=x)$ per month. The other information associated to the Inventory management is given below:

The set up cost per cycle $A=\$ 350$, unit holding cost for perfect items $h_{w}=\$ 3.5 / \$ /$ month, unit holding cost for scrap items $h_{s}=\$ 1.5 / \$ /$ month, unit selling price $s=\$ 50$, unit inspection cost $d_{i}=\$ 0.5$, unit salvage value $w=\$ 5$, unit purchasing cost $c=\$ 25$, Also, expected value of percentage rate of scrap items and reworkable items are $E\left(r_{s}\right)=0.02, E\left(r_{w}\right)=0.06$ respectively. The variance $E\left(1-r_{s}\right)^{2}=0.9$. Now our problem is to find the best order quantity $Q^{*}$ and the optimum cycle time $T^{*}$ such that the DM will win maximum monthly total profit $Z_{E}^{*}$ of the crisp case. This is obtained that: $Q^{*}=909.628$ units, $=\$ 23296.40$ and the cycle time is $T^{*}=10.697$ months. We also compute the numerical solutions of the fuzzy as well as dense fuzzy models and obtain the expected profit which is shown in Tables 1 and 2.

The Table 1 shows, in the case of general fuzzy solution if the left deviation assumes greater value than right deviation then the expected profit becomes $\$ 22700$ but for its reverse it gets maximum value to $\$ 23869$. If both the deviations are same then the profit value so obtained might lie in between them. Here also we see that, lower left fuzzy deviation (with respect to right deviation) giving higher profit value than crisp optimum, the other cases give lower profit optimum than crisp optimum as a whole. Table 2 shows that for the case of dense fuzzy model, the lower left fuzzy deviations giving a sudden downwards jump/suicidal jump of the expected profit function tremendously. But, for greater left fuzzy deviations we see the profit value reaches to maximum height. However, for equal fuzzy deviations, the profit value lies in between the above results.

\subsection{Sensitivity analysis of the crisp and dense fuzzy model}

For better justification of the proposed model we need to make the sensitivity analysis for the crisp as well as dense fuzzy model. To do this we take the changes from $-50 \%$ to $+50 \%$ of all the parametric values associated with the crisp model (shown in Tab. 3) and for the dense fuzzy model we perform that changes of the fuzzy deviations $(\rho, \sigma)$ by means of vertices of a unit square only and taking the help of LINGO software, the numerical results are put in the Tables 4, 5, 6 and 7, respectively. 
TABLE 3. Sensitivity analysis for crisp model.

\begin{tabular}{|c|c|c|c|c|c|}
\hline Parametri & change & $T^{*}$ & $Q^{*}$ & $Z_{E}^{*}$ & $R_{z}=\left\{\frac{Z_{E}^{*}-Z_{E}^{*}-\operatorname{crisp}}{Z_{E}^{*}-\operatorname{crisp}}\right\} \times 100 \%$ \\
\hline \multirow[t]{4}{*}{$A$} & $+50 \%$ & 13.103 & 1114.062 & 23119.90 & -0.76 \\
\hline & $+30 \%$ & 12.197 & 1037.135 & 23186.31 & -0.47 \\
\hline & $-30 \%$ & 8.950 & 761.049 & 23424.65 & +0.55 \\
\hline & $-50 \%$ & 7.564 & 643.204 & 23526.38 & +0.98 \\
\hline \multirow[t]{4}{*}{$D$} & $+50 \%$ & 7.352 & 937.762 & 34979.91 & +50.15 \\
\hline & $+30 \%$ & 8.402 & 928.828 & 30306.40 & +30.09 \\
\hline & $-30 \%$ & 14.732 & 876.920 & 16286.97 & -30.09 \\
\hline & $-50 \%$ & 19.718 & 838.338 & 11614.81 & -93.06 \\
\hline \multirow[t]{4}{*}{$x$} & $+50 \%$ & 12.557 & 1067.738 & 23412.66 & +0.49 \\
\hline & $+30 \%$ & 11.885 & 1010.606 & 23374.84 & +0.34 \\
\hline & $-30 \%$ & 9.918 & 782.135 & 23168.38 & -0.55 \\
\hline & $-50 \%$ & 7.923 & 673.762 & 23021.49 & -1.18 \\
\hline \multirow[t]{4}{*}{$s$} & $+50 \%$ & 10.697 & 909.628 & 48296.38 & +107.3 \\
\hline & $+30 \%$ & 10.697 & 909.628 & 38296.38 & +64.38 \\
\hline & $-30 \%$ & 10.697 & 909.628 & 8296.38 & -64.38 \\
\hline & $-50 \%$ & \multicolumn{3}{|c|}{ No feasible solution } & \\
\hline \multirow[t]{4}{*}{$W$} & $+50 \%$ & 10.697 & 909.628 & 23347.40 & +0.22 \\
\hline & $+30 \%$ & 10.697 & 909.628 & 23326.99 & +0.13 \\
\hline & $-30 \%$ & 10.697 & 909.628 & 23265.77 & -0.13 \\
\hline & $-50 \%$ & 10.697 & 909.628 & 23245.36 & -0.22 \\
\hline \multirow[t]{4}{*}{$d_{i}$} & $+50 \%$ & 10.697 & 909.628 & 23041.30 & -1.09 \\
\hline & $+30 \%$ & 10.697 & 909.628 & 23143.32 & -0.65 \\
\hline & $-30 \%$ & 10.697 & 909.628 & 23449.44 & +0.65 \\
\hline & $-50 \%$ & 10.697 & 909.628 & 23551.48 & +1.09 \\
\hline \multirow[t]{3}{*}{$h_{w}$} & $+50 \%$ & 5.914 & 502.927 & 22661.38 & -2.73 \\
\hline & $+30 \%$ & 6.959 & 591.771 & 22874.60 & -1.81 \\
\hline & $\begin{array}{l}-30 \% \\
-50 \%\end{array}$ & \multicolumn{3}{|c|}{ No feasible solution } & \\
\hline \multirow[t]{4}{*}{$h_{s}$} & $+50 \%$ & 10.688 & 908.823 & 23295.69 & -0.0003 \\
\hline & $+30 \%$ & 10.691 & 909.144 & 23295.96 & -0.0001 \\
\hline & $-30 \%$ & 10.703 & 910.110 & 23296.80 & +0.002 \\
\hline & $-50 \%$ & 10.707 & 910.436 & 23297.08 & +0.003 \\
\hline \multirow[t]{4}{*}{$c$} & $+50 \%$ & \multirow{2}{*}{\multicolumn{3}{|c|}{ No feasible solution }} & \\
\hline & $+30 \%$ & & & & \\
\hline & $-30 \%$ & 7.446 & 633.179 & 30606.61 & +31.38 \\
\hline & $-50 \%$ & 6.423 & 546.242 & 35529.10 & +52.50 \\
\hline \multirow[t]{4}{*}{$E\left(r_{s}\right)$} & $+50 \%$ & 10.601 & 910.705 & 23073.59 & -0.95 \\
\hline & $+30 \%$ & 10.639 & 910.274 & 23163.26 & -0.57 \\
\hline & $-30 \%$ & 10.755 & 908.983 & 23427.90 & +0.56 \\
\hline & $-50 \%$ & 10.794 & 908.555 & 23514.69 & +0.94 \\
\hline \multirow[t]{4}{*}{$E\left(r_{w}\right)$} & $+50 \%$ & \multirow{2}{*}{\multicolumn{3}{|c|}{ No feasible solution }} & \\
\hline & $+30 \%$ & & & & \\
\hline & $-30 \%$ & 7.446 & 633.179 & 22953.54 & -1.47 \\
\hline & $-50 \%$ & 6.423 & 546.242 & 22774.00 & -2.24 \\
\hline \multirow[t]{3}{*}{$E\left(1-r_{s}\right)$} & $+50 \%$ & 6.323 & 537.714 & 22753.26 & -2.33 \\
\hline & $+30 \%$ & 7.352 & 625.174 & 22939.09 & -1.53 \\
\hline & $\begin{array}{l}-30 \% \\
-50 \%\end{array}$ & \multicolumn{3}{|c|}{ No feasible solution } & \\
\hline
\end{tabular}


TABLE 4. Sensitivity analysis at $(\rho, \sigma)=(0.2,0.3)$.

\begin{tabular}{|c|c|c|c|c|c|c|}
\hline$n$ & Parametric change & $I\left(Q^{*}\right)$ & $I\left(T^{*}\right)$ & $I\left(Z_{E}^{*}\right)$ & $R_{z}(\%)$ & $R_{Q}(\%)$ \\
\hline \multirow{7}{*}{1} & \multirow[t]{4}{*}{$50 \%$} & 1739.498 & 21.1 & 43833.32 & 88.15 & 91.23 \\
\hline & & 1747.310 & 21.9 & 42206.69 & 81.17 & 92.09 \\
\hline & & \multirow{2}{*}{\multicolumn{5}{|c|}{ No feasible solution }} \\
\hline & & & & & & \\
\hline & $\begin{array}{ll}\sigma & +50 \%\end{array}$ & \multicolumn{5}{|c|}{ No feasible solution } \\
\hline & $\begin{array}{l}+30 \% \\
-30 \%\end{array}$ & 1764.669 & 21.0 & 42958.33 & 84.4 & 93.99 \\
\hline & $-50 \%$ & 1769.291 & 21.2 & 44323.28 & 90.25 & 94.51 \\
\hline
\end{tabular}

Notes. Where, $R_{z}=\left\{\frac{I\left(Z_{E}^{*}\right)-Z_{E}^{*}}{Z_{E}^{*}}\right\} \times 100 \%$ and $R_{Q}=\left\{\frac{I\left(Q^{*}\right)-Q^{*}}{Q^{*}}\right\} \times 100 \%$.

TABle 5. Sensitivity Analysis at $(\rho, \sigma)=(0.2,0.2)$.

\begin{tabular}{|c|c|c|c|c|c|c|}
\hline$n$ & Parametric change & $I\left(Q^{*}\right)$ & $I\left(T^{*}\right)$ & $I\left(Z_{E}^{*}\right)$ & $R_{z}(\%)$ & $R_{Q}(\%)$ \\
\hline \multirow{8}{*}{1} & $+50 \%$ & 1746.186 & 21.4 & 44156.25 & 89.54 & 91.96 \\
\hline & $+30 \%$ & 1754.000 & 21.2 & 44250.37 & 89.95 & 92.82 \\
\hline & $-30 \%$ & \multirow{2}{*}{\multicolumn{5}{|c|}{ No feasible solution }} \\
\hline & $-50 \%$ & & & & & \\
\hline & $+50 \%$ & 1758.713 & 20.7 & 1417.28 & -93.92 & 93.34 \\
\hline & $+30 \%$ & 1761.224 & 20.8 & 28638.43 & 22.94 & 93.62 \\
\hline & $-30 \%$ & 1770.117 & 21.3 & 44365.28 & 90.44 & 94.59 \\
\hline & $-50 \%$ & 1773.590 & 21.4 & 44261.13 & 89.99 & 94.97 \\
\hline
\end{tabular}

Notes. Where, $R_{z}=\left\{\frac{I\left(Z_{E}^{*}\right)-Z_{E}^{*}}{Z_{E}^{*}}\right\} \times 100 \%$ and $R_{Q}=\left\{\frac{I\left(Q^{*}\right)-Q^{*}}{Q^{*}}\right\} \times 100 \%$.

TABLE 6. Sensitivity analysis at $(\rho, \sigma)=(0.3,0.2)$.

\begin{tabular}{|c|c|c|c|c|c|c|}
\hline$n$ & Parametric change & $I\left(Q^{*}\right)$ & $I\left(T^{*}\right)$ & $I\left(Z_{E}^{*}\right)$ & $R_{z}(\%)$ & $R_{Q}(\%)$ \\
\hline \multirow{8}{*}{1} & $\rho \quad+50 \%$ & 1715.110 & 22.1 & 43004.79 & 84.59 & 88.55 \\
\hline & $+30 \%$ & 1727.909 & 21.8 & 43534.84 & 86.87 & 89.95 \\
\hline & $-30 \%$ & 1763.526 & 21.1 & 43750.55 & 87.79 & 93.87 \\
\hline & $-50 \%$ & \multicolumn{5}{|c|}{ No feasible solution } \\
\hline & $+50 \%$ & 1739.498 & 21.1 & 43833.13 & 88.15 & 91.23 \\
\hline & $+30 \%$ & 1742.009 & 21.2 & 44161.58 & 89.56 & 91.50 \\
\hline & $-30 \%$ & 1750.902 & 21.6 & 43764.60 & 87.86 & 92.48 \\
\hline & $-50 \%$ & 1754.375 & 21.7 & 43242.30 & 85.62 & 92.86 \\
\hline
\end{tabular}

Notes. Where, $R_{z}=\left\{\frac{I\left(Z_{E}^{*}\right)-Z_{E}^{*}}{Z_{E}^{*}}\right\} \times 100 \%$ and $R_{Q}=\left\{\frac{I\left(Q^{*}\right)-Q^{*}}{Q^{*}}\right\} \times 100 \%$.

\subsection{Discussion on sensitivity Tables $3-7$}

Sensitivity analysis Table 3 reveals that, among 12 several parameters, only the monthly demand $D$ and unit selling price $s$ are highly sensitive parameters. The other parameters are more or less insensitive with respect to the initial crisp value. At $+50 \%$ change of $D$ and $s$, the expected profits reach to $\$ 34979.91$ and $\$ 48296.38$, respectively. Moreover, at $-50 \%$ changes of the parameter $D$ the expected profit assumes value $\$ 11614.81$ which is the reduction of $93.06 \%$ and that for swe get no feasible solution. Table 4 shows that for the case of $\rho$ at 
TABLE 7 . Sensitivity analysis at $(\rho, \sigma)=(0.3,0.3)$.

\begin{tabular}{|c|c|c|c|c|c|c|}
\hline$n$ & Parametric change & $I\left(Q^{*}\right)$ & $I\left(T^{*}\right)$ & $I\left(Z_{E}^{*}\right)$ & $R_{z}(\%)$ & $R_{Q}(\%)$ \\
\hline \multirow{8}{*}{1} & \multirow[t]{4}{*}{$\rho$} & 1708.422 & 21.84 & 43811.82 & 88.06 & 87.89 \\
\hline & & 1721.220 & 21.50 & 44124.23 & 89.41 & 89.33 \\
\hline & & \multirow{2}{*}{\multicolumn{5}{|c|}{ No feasible solution }} \\
\hline & & & & & & \\
\hline & $+50 \%$ & \multicolumn{5}{|c|}{ No feasible solution } \\
\hline & $+30 \%$ & 1734.567 & 20.82 & 38796.96 & 66.54 & 90.76 \\
\hline & $-30 \%$ & 1745.454 & 21.36 & 44186.28 & 89.67 & 91.97 \\
\hline & $-50 \%$ & 1750.076 & 21.58 & 43856.53 & 88.26 & 92.52 \\
\hline
\end{tabular}

Notes. Where, $R_{z}=\left\{\frac{I\left(Z_{E}^{*}\right)-Z_{E}^{*}}{Z_{E}^{*}}\right\} \times 100 \%$ and $R_{Q}=\left\{\frac{I\left(Q^{*}\right)-Q^{*}}{Q^{*}}\right\} \times 100 \%$.

$-30 \%,-50 \%$ and for the case of $\sigma$ at $+50 \%,+30 \%$ the objective function giving no feasible solutions, but for the other cases the expected profit getting the bounds $(\$ 42206.69, \$ 44323.28)$ by reaching the enhancement $+81-92 \%$ approx with respect to the increase of crisp order quantity and cycle time by $+91-95 \%$ approx. In Table 5, we see a suicidal jump of the expected profit function occurs at $+50 \%$ change of $\sigma$ by reducing the value to $-93.92 \%$ and at $-30 \%,-50 \%$ changes of $\rho$ no feasible solution occurs. For the other cases the profit lies within the bounds $(\$ 28638.43, \$ 44365.28)$. Table 6 shows that at $-50 \%$ change of $\rho$ the objective function has no feasible solution, but for the other cases the profit value assumes the bounds ( $\$ 43004.79, \$ 44161.58$ ) by the crisp enhancement $+84-89 \%$ alone. Table 7 shows that at $-50 \%,-30 \%$ changes of $\rho$ and that for $\sigma$ at $+50 \%$ the objective function has no feasible solution, but beyond those the profit value assumes the bounds $(\$ 43811.82, \$ 44186.28)$ by the crisp enhancement $+66-89 \%$ alone. However, throughout the whole Tables $4-7$ we see that the cycle time as well as order quantity jump tremendously to provide the maximum profit except the suicidal jump.

\section{Graphical illustrations}

Here we draw different graphs based on table data to justify the model. Figure 3 shows the specific overview of the profit function under dense fuzzy environment. It shows whenever we are considering the crisp as well as the fuzzy environment for optimizing the objective function we are getting very close solution but at the dense fuzzy environment the expected profit assumes almost double of that crisp/fuzzy solutions. Figure 3 reveals that, the model optimum exists and it follows the edges of a $(\rho, \sigma)$ paradoxical square. At minimum $\sigma-$ path [referring to the coordinate $(0.2,0.1)$ ] the highest profit value lies having coordinate $(0.2,0.14)]$ amounting $\$ 44365.28$. Then it goes towards right handed upper edges getting zigzag values. The top left vertex shows the suicidal [see Appendix A.4] point in which a great loss of profit occurs. Beyond the paradoxical path the profit values getting decreasing. Figure 4 shows the general overview of the expected profit function under $(\rho, \sigma)$ paradoxical coordinates. The four coordinate points constitutes a small square unit called vertices of that unit square. If we go across the vertical line on $\sigma$-axis keeping $\rho$ value at minimum then the expected profit quickly jumps down to minimum value. But if we would like to pass across the $\rho$-axis keeping the $\sigma$ value at minimum then the expected profit reaches to the highest value. Again if we think of $(\rho, \sigma)$ at lower and upper vertex of the unit square then we see fuzzy solutions began to decrease but the dense fuzzy solutions getting jumps to higher values respectively (Fig. 5).

\section{CONCLUSion}

In this article we have developed a profit seeking EOQ model for imperfect quality items with receiving reparative batch and order overlapping under dense fuzzy environment. To avoid the shortages, the orders are 


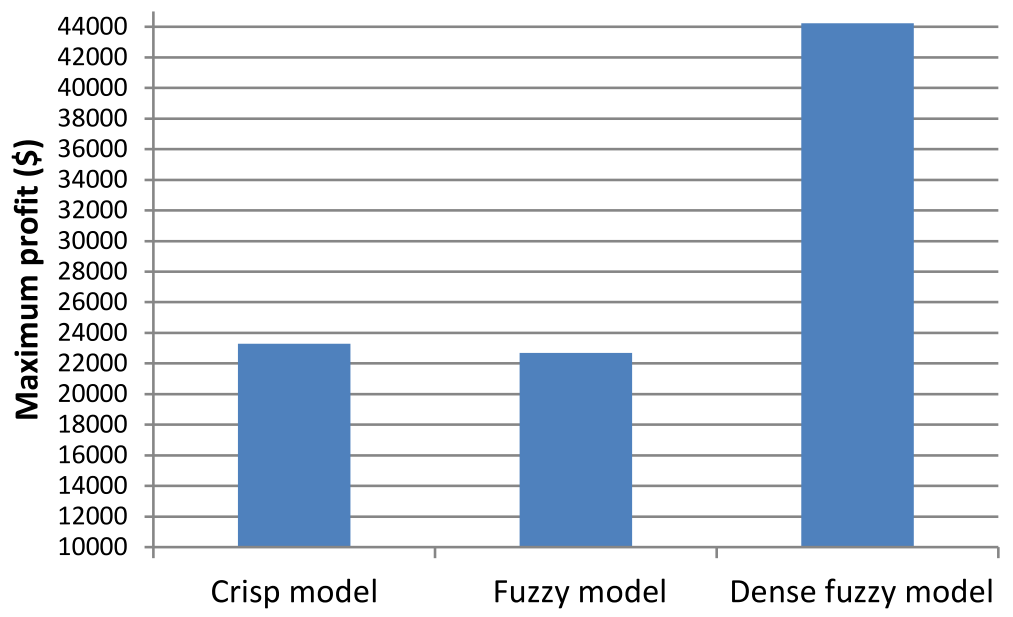

FiguRE 3. Optimal profit under several environments.

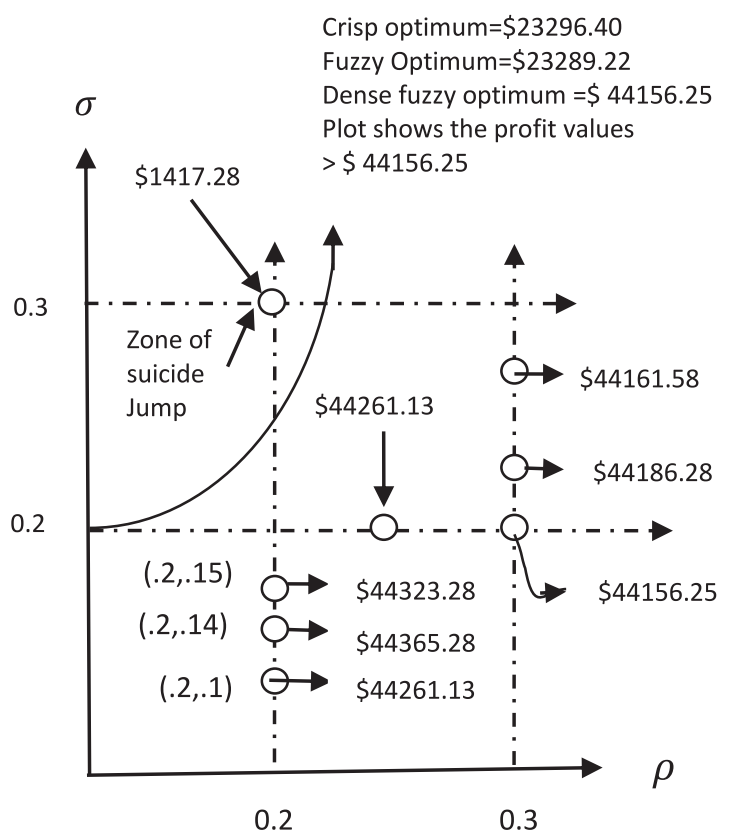

FIgURE 4. Optimum profit path at paradoxical square.

overlapped for customers' satisfactions, scrap items are separated through screening process and a salvage value has been taking place for that scrap items. At the end of the cycle time a profit function is developed in which the order quantity is the decision variable. As per real situations, since the demand quantity per month may vary with learning experiences of the decision maker (DM) over interaction/negotiations with the learned customers, so it is quiet natural to consider the monthly demand quantity as dense fuzzy number. The basic overview of the dense fuzzy number is to reduce the uncertainty on demand per cycle. By this way, it will be quite easier to any DM so that (s)he could order the specific requirements of the customers without hesitation. Defuzzification is done with the help of ranking index rule for both fuzzy and dense fuzzy models. This study explores that 

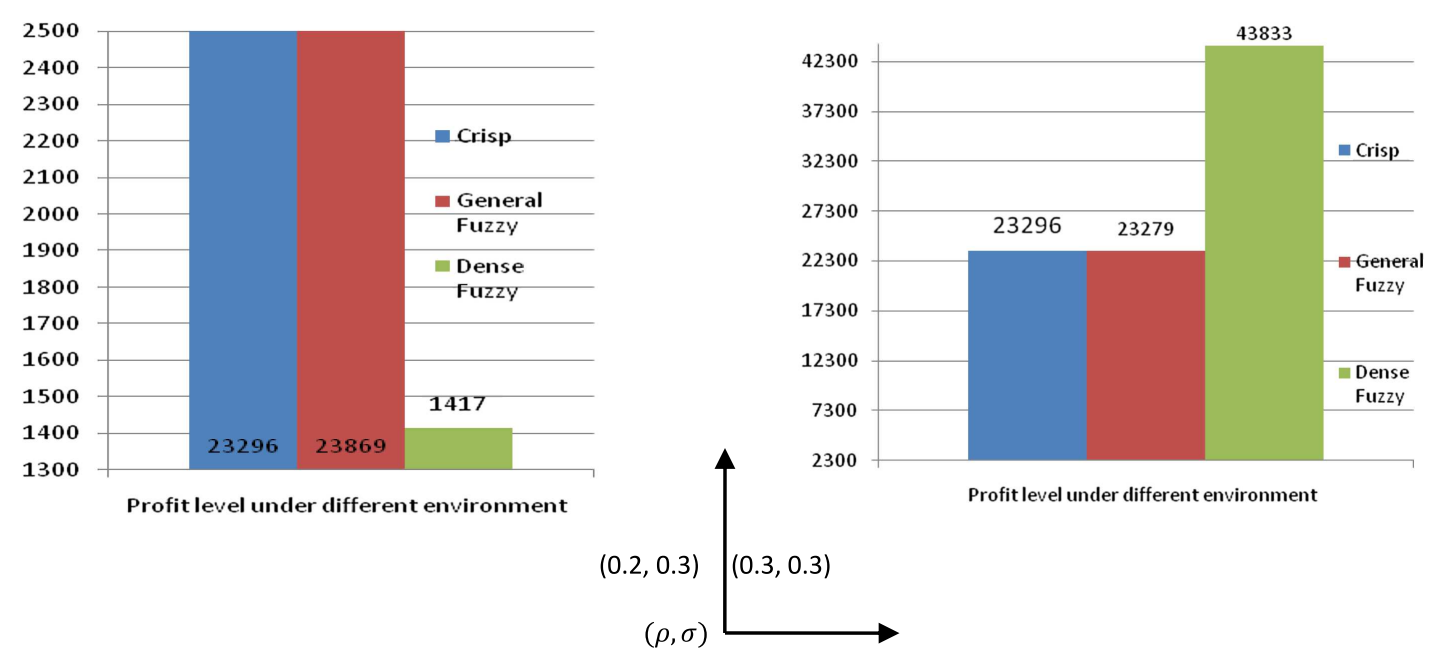

$(0.2,0.2) \quad(0.3,0.2)$
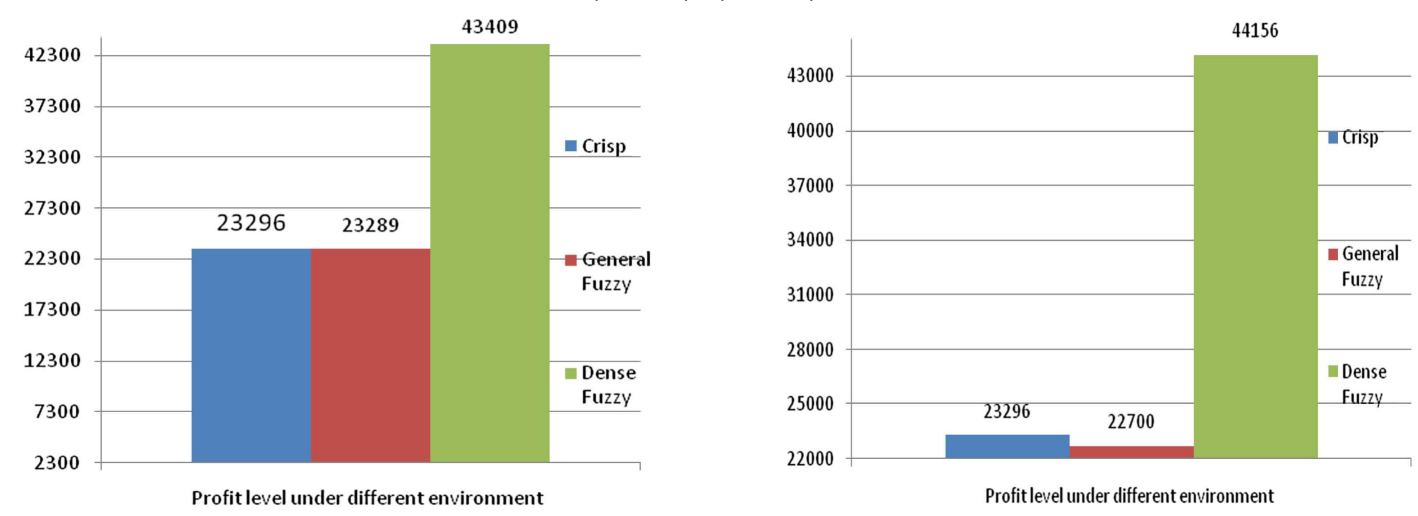

FiguRE 5. Profit Jump under $(\rho, \sigma)$ paradoxical coordinates.

a tremendous situation exists to reach into a golden profit which is beyond the imagination of crisp, even the general fuzzy environment also. However, a situation may come where huge amount of profit loss arises. We rename this situation as the suicide zone. Moreover, we call the profit jump against the $(\rho, \sigma)$-coordinate as paradoxical because, based on four vertical co-ordinates of the unit square if we wish to get a profit sensitivity then we might have seen that the zone of suicide point and the zone of golden point must lie on the boundary of the same square or any one of its foot. To overcome this zone, the DM might have to follow the unit square paradoxical alert during experiencing the inventory management process exclusively.

\section{Scope of future work}

Considering all the cost coefficients as dense fuzzy or intuitionistic dense fuzzy numbers to the related models of this study anyone can develop more new research in these directions. 


\section{Appendix A.}

\section{A.1. Basic arithmetic operations on triangular fuzzy number (TFN)}

Let $A=\left\langle a_{1}, a_{2}, a_{3}\right\rangle$ and $B=\left\langle b_{1}, b_{2}, b_{3}\right\rangle$ be two TFN, then for usual Arithmetic operations $\{+,-, \times, \div\}$, namely addition, subtraction, multiplication between $A$ and $B$ are given below

(i) $A+B=\left\langle a_{1}+b_{1}, a_{2}+b_{2}, a_{3}+b_{3}\right\rangle$,

(ii) $A-B=\left\langle a_{1}-b_{3}, a_{2}-b_{2}, a_{3}-b_{1}\right\rangle$,

(iii) $\mathrm{A} \times B=\left\langle\operatorname{Min}\left(a_{i} b_{j}\right), \operatorname{Max}\left(a_{i} b_{j}\right)\right\rangle \forall i, j=1,2,3$,

(iv) $A / B=\left\langle\operatorname{Min}\left(a_{\mathrm{i}} / b_{\mathrm{j}}\right), \operatorname{Max}\left(a_{\mathrm{i}} / b_{\mathrm{j}}\right)\right\rangle$ for $b_{j} \neq 0, \forall i, j=1,2,3$,

(v) $\begin{aligned} \delta A & =\left\langle\delta a_{1}, \delta a_{2}, \delta a_{3}\right\rangle & & \text { if } \delta \geq 0 \\ \text { and } \delta A & =\left\langle\delta a_{3}, \delta a_{2}, \delta a_{1}\right\rangle & & \text { if } \delta<0\end{aligned}$.

\section{A.2. Crisp convergence for the objective function}

First of all we shall transfer the dense fuzzy into general fuzzy by putting $n=0$ throughout, then taking limit as under $(\rho \rightarrow 0 \leftarrow \sigma)$. It is seen that, as $\rho \rightarrow 0 \leftarrow \sigma$ then

$$
D_{1}=D_{2}\left(1-\frac{\rho}{1+n}\right) \rightarrow D_{2} \quad \text { and } \quad D_{3}=D_{2}\left(1+\frac{\sigma}{1+n}\right) \rightarrow D_{2} .
$$

Moreover we have $Q_{1}=\sqrt{\frac{2 A D_{2}\left(1-\frac{\rho}{1+n}\right)}{D_{2} Y_{1}+Y_{2}}} \rightarrow \sqrt{\frac{2 A D_{2}}{D_{2} Y_{1}+Y_{2}}} ; \quad Q_{2}=\sqrt{\frac{2 A D_{2}}{D_{2} Y_{1}\left(1+\frac{\sigma}{1+n}\right)+Y_{2}}} \rightarrow \sqrt{\frac{2 A D_{2}}{D_{2} Y_{1}+Y_{2}}}$ and $Q_{3}=$ $\sqrt{\frac{2 A D_{2}\left(1+\frac{\sigma}{1+n}\right)}{D_{2} Y_{1}+Y_{2}}} \rightarrow \sqrt{\frac{2 A D_{2}}{D_{2} Y_{1}+Y_{2}}}$. Thus from above, $D_{1} \rightarrow D_{2} \leftarrow D_{3} \Rightarrow D_{1} \rightarrow D \leftarrow D_{3}$ and $Q_{3} \rightarrow Q_{1} \leftarrow Q_{2} \Rightarrow$ $\sqrt{\frac{2 A D}{D Y_{1}+Y_{2}}}=Q$.

So,

$$
\begin{aligned}
\lim _{\substack{D_{1} \rightarrow D_{2} \\
\dot{D}_{3} \rightarrow D_{2}}} I\left(Z_{E}^{*}\right)= & \frac{4 D_{2} F_{1}}{4}-\frac{F_{4}}{4}\left(Q_{1}+2 Q_{2}+Q_{3}\right)-\frac{D_{2} F_{3}}{12}\left[Q_{3}+Q_{2}+2 Q_{3}+4 Q_{2}+2 Q_{1}+Q_{1}+Q_{2}\right] \\
& -\frac{D_{2} F_{2}}{2}\left[\frac{Q_{2}-Q_{1}}{\left(Q_{2}-Q_{1}\right)} \log \left(\frac{Q_{2}}{Q_{1}}\right)-\frac{Q_{3}-Q_{2}}{\left(Q_{3}-Q_{2}\right)} \log \left(\frac{Q_{2}}{Q_{3}}\right)\right. \\
& \left.-\frac{Q_{3}+2 Q_{2}+Q_{1}-Q_{1}-Q_{2}-Q_{3}-Q_{2}}{Q_{1} Q_{2}+Q_{3} Q_{2}-Q_{3} Q_{1}-Q_{2}^{2}}\right]
\end{aligned}
$$

and that for order quantity is given by $I\left(Q^{*}\right)=\frac{1}{4}\left(Q_{1}+2 Q_{2}+Q_{3}\right)$

$$
\begin{aligned}
\Rightarrow & \lim _{\substack{Q_{1} \rightarrow Q_{2} \\
\dot{Q}_{3} \rightarrow \dot{Q}_{2} \\
\dot{S}^{\prime}}} I\left(Z_{E}^{*}\right)=D_{2} F_{1}-Q_{2} F_{4}-D_{2} Q_{2} F_{3} \\
& -\frac{D_{2} F_{2}}{2} \lim _{\substack{Q_{1} \rightarrow Q_{2} \\
\dot{Q}_{3} \rightarrow \dot{Q}_{2}}}\left[\frac{\log \left(\frac{Q_{2}}{Q_{1}}\right)}{\left(Q_{2}-Q_{1}\right)}-\frac{\log \left(\frac{Q_{2}}{Q_{3}}\right)}{\left(Q_{3}-Q_{2}\right)}-\frac{Q_{3}+2 Q_{2}+Q_{1}-Q_{1}-Q_{2}-Q_{3}-Q_{2}}{Q_{1} Q_{2}+Q_{3} Q_{2}-Q_{3} Q_{1}-Q_{2}^{2}}\right]
\end{aligned}
$$




$$
\begin{aligned}
& \Rightarrow \lim _{\substack{Q_{1} \rightarrow Q_{2} \\
\dot{Q}_{3} \rightarrow Q_{1}}} I\left(Z_{E}^{*}\right)=D_{2} F_{1}-Q_{2} F_{4}-D_{2} Q_{2} F_{3}-\frac{D_{2} F_{2}}{2} \lim _{\substack{Q_{1} \rightarrow Q_{1} \\
\dot{Q}_{3} \rightarrow \dot{Q}_{2}}}\left[\frac{\log \left(\frac{Q_{2}}{Q_{1}}\right)}{\left(Q_{2}-Q_{1}\right)}-\frac{\log \left(\frac{Q_{2}}{Q_{3}}\right)}{\left(Q_{3}-Q_{2}\right)}\right] \\
& \Rightarrow \lim _{\substack{Q_{1} \rightarrow Q_{2} \\
Q_{3}^{\prime} \rightarrow Q_{1}^{\prime}}} I\left(Z_{E}^{*}\right)=D_{2} F_{1}-Q_{2} F_{4}-D_{2} Q_{2} F_{3}-\frac{D_{2} F_{2}}{2 Q_{2}} \lim _{\substack{Q_{1} \rightarrow Q_{2} \\
Q_{3} \rightarrow Q_{1}}}\left[\frac{\log \left(\frac{Q_{2}}{Q_{1}}\right)}{\left(1-\frac{Q_{1}}{Q_{2}}\right)}-\frac{\log \left(\frac{Q_{2}}{Q_{3}}\right)}{\left(\frac{Q_{3}}{Q_{2}}-1\right)}\right] \\
& =D_{2} F_{1}-Q_{2} F_{4}-D_{2} Q_{2} F_{3}-\frac{D_{2} F_{2}}{2 Q_{2}} \lim _{\substack{Q_{1} \rightarrow Q_{2} \\
\dot{Q}_{3} \rightarrow Q_{1}}}\left[\frac{\log \left(\frac{Q_{2}}{Q_{1}}\right)}{\left(1-\frac{Q_{1}}{Q_{2}}\right)}+\frac{\log \left(\frac{Q_{2}}{Q_{1}}\right)}{\left(1-\frac{Q_{1}}{Q_{2}}\right)}\right] \\
& =D_{2} F_{1}-Q_{2} F_{4}-D_{2} Q_{2} F_{3}-\frac{D_{2} F_{2}}{Q_{2}} \lim _{Q_{1} \rightarrow Q_{2}}\left[\frac{\log \left(\frac{Q_{2}}{Q_{1}}\right)}{\left(1-\frac{Q_{1}}{Q_{2}}\right)}\right] \\
& =D_{2} F_{1}-Q_{2} F_{4}-D_{2} Q_{2} F_{3}-\frac{D_{2} F_{2}}{Q_{2}} \lim _{Q_{1} \rightarrow Q_{2}}\left[\frac{\log \left(\frac{Q_{1}}{Q_{2}}\right)}{\left(\frac{Q_{1}}{Q_{2}}-1\right)}\right] \\
& =D_{2} F_{1}-Q_{2} F_{4}-D_{2} Q_{2} F_{3}-\frac{D_{2} F_{2}}{Q_{2}} \lim _{\frac{Q_{1}}{Q_{2}} \rightarrow 1}\left[\frac{\log \left(\frac{Q_{1}}{Q_{2}}-1+1\right)}{\left(\frac{Q_{1}}{Q_{2}}-1\right)}\right] \\
& =D_{2} F_{1}-Q_{2} F_{4}-D_{2} Q_{2} F_{3}-\frac{D_{2} F_{2}}{Q_{2}} \lim _{k \rightarrow 0}\left[\frac{\log (k+1)}{k}\right], \text { for } \frac{Q_{1}}{Q_{2}}-1=k \text { as } \frac{Q_{1}}{Q_{2}} \rightarrow 1, k \rightarrow 0 \\
& =D_{2} F_{1}-Q_{2} F_{4}-D_{2} Q_{2} F_{3}-\frac{D_{2} F_{2}}{Q_{2}} \\
& \Rightarrow I\left(Z_{E}^{*}\right)=D F_{1}-Q^{*} F_{4}-D Q^{*} F_{3}-\frac{D F_{2}}{Q^{*}},\left[\text { Since, } D_{2}=D, Q_{2} \rightarrow Q^{*}\right] \text {. }
\end{aligned}
$$

Because, $Q_{2}=\sqrt{\frac{2 A D_{2}}{D_{2} Y_{1}(1+\sigma)+Y_{2}}} \rightarrow \sqrt{\frac{2 A D}{D Y_{1}+Y_{2}}} \rightarrow Q^{*}$ as $\sigma \rightarrow 0$.

Hence we arrive at the crisp objectives function.

\section{A.3. Crisp convergence for the cycle time $T$}

We consider the expected time equation (4.18) and taking limits on it as follows:

$$
\begin{aligned}
& \lim _{\substack{Q_{1} \rightarrow Q_{2} \\
\dot{Q}_{3} \rightarrow \dot{Q}_{2}}} I\left(T^{*}\right)=\frac{\left(1-r_{s}\right)}{2}\left[\frac{D_{2} Q_{3}-D_{1} Q_{2}}{\left(D_{2}-D_{1}\right)^{2}} \log \left(\frac{D_{2}}{D_{1}}\right)-\frac{D_{3} Q_{2}-D_{2} Q_{1}}{\left(D_{3}-D_{2}\right)^{2}} \log \left(\frac{D_{2}}{D_{3}}\right)\right. \\
& \left.-\frac{D_{3} Q_{3}+2 Q_{2} D_{2}+Q_{1} D_{1}-D_{1} Q_{2}-D_{2} Q_{1}-D_{3} Q_{2}-Q_{2} D_{3}}{D_{1} D_{2}+D_{3} D_{2}-D_{3} D_{1}-D_{2}^{2}}\right] \\
& =\lim _{\substack{D_{1} \rightarrow D_{2} \\
\dot{D}_{3} \rightarrow D_{1}}} \frac{\left(1-r_{s}\right) Q_{2}}{2}\left[\frac{D_{2}-D_{1}}{\left(D_{2}-D_{1}\right)} \log \left(\frac{D_{2}}{D_{1}}\right)-\frac{D_{3}-D_{2}}{\left(D_{3}-D_{2}\right)} \log \left(\frac{D_{2}}{D_{3}}\right)\right]
\end{aligned}
$$




$$
\begin{aligned}
& =\lim _{\substack{D_{1} \rightarrow D_{2} \\
D_{3} \rightarrow D_{1}}} \frac{\left(1-r_{s}\right) Q_{2}}{2}\left[\frac{\log \left(\frac{D_{2}}{D_{1}}\right)}{\left(D_{2}-D_{1}\right)}-\frac{\log \left(\frac{D_{2}}{D_{3}}\right)}{\left(D_{3}-D_{2}\right)}\right] \\
& =\lim _{\substack{D_{1} \rightarrow D_{2} \\
D_{3} \rightarrow D_{1}}} \frac{\left(1-r_{s}\right) Q_{2}}{2}\left[\frac{\log \left(\frac{D_{2}}{D_{1}}\right)}{\left(D_{2}-D_{1}\right)}+\frac{\log \left(\frac{D_{2}}{D_{1}}\right)}{\left(D_{2}-D_{1}\right)}\right] \\
& =\lim _{\substack{D_{1} \rightarrow D_{2} \\
\dot{D}_{3} \rightarrow \dot{D}_{1}}} \frac{\left(1-r_{s}\right) Q_{2}}{D_{2}}\left[\frac{\log \left(\frac{D_{2}}{D_{1}}\right)}{\left(1-\frac{D_{1}}{D_{2}}\right)}\right]=\lim _{D_{1} \rightarrow D_{2}} \frac{-\left(1-r_{s}\right) Q_{2}}{D_{2}}\left[\frac{\log \left(\frac{D_{2}}{D_{1}}\right)}{\left(\frac{D_{1}}{D_{2}}-1\right)}\right] \\
& =\lim _{\substack{D_{1} \rightarrow D_{2} \\
,}} \frac{\left(1-r_{s}\right) Q_{2}}{D_{2}}\left[\frac{\log \left(\frac{D_{2}}{D_{1}}\right)}{\left(\frac{D_{1}}{D_{2}}-1\right)}\right] \\
& =\lim _{\substack{D_{1} \\
D_{2}}} \frac{\left(1-r_{s}\right) Q_{2}}{D_{2}}\left[\frac{\log \left(1+\frac{D_{1}}{D_{2}}-1\right)}{\left(\frac{D_{1}}{D_{2}}-1\right)}\right]=\frac{\left(1-r_{s}\right) Q_{2}}{D_{2}} \Rightarrow T^{*}=\frac{\left(1-r_{s}\right) Q^{*}}{D} .
\end{aligned}
$$

\section{A.4. Suicidal point}

A tremendous loss of profit occurs due to careless attempt/drive or decision of inventory practitioner (decision maker). A story behind it, in Bidarva, the district of Maharastra, India, whenever the cotton producing farmers fall into great loss of their production due to natural calamity or careless use of pesticides in the cotton field they usually get suicide in that field to escape themselves from the larger amount of bank loans and from the pressures of livelihoods of their family.

Acknowledgements. The authors are thankful to the anonymous reviewers, honorable Associate Editor and Editor-in Chief for their valuable suggestions to improve the quality of this article.

Conflicts of interest. The authors declare that they have no conflicts of interest regarding the publication of the article.

\section{REFERENCES}

[1] T. Allahviranloo and R. Saneifard, Defuzzification method for ranking fuzzy numbers based on center of gravity. Iran. J. Fuzzy Syst. 9 (2012) 57-67.

[2] R.E. Bellman and L.A. Zadeh, Decision making in a fuzzy environment. Manage. Sci. 17 (1970) B141-B164.

[3] R. Cerulli, C. D'Ambrosio and M. Gentili, Best and worst values of the optimal cost of the interval transportation problem. ODS 2017: Optimization and Decision Science: Methodologies and Applications. In Vol. 217 of Springer Proceedings in Mathematics 83 Statistics. Springer (2017) 367-374.

[4] H.C. Chang, An application of fuzzy sets theory to the EOQ model with imperfect quality items. Comput. Oper. Res. 31 (2004) 2079-2092.

[5] S.C. Chang, J.S. Yao and H.M. Lee, Economic reorder point for fuzzy backorder quantity. Eur. J. Oper. Res. 109 (1998) $183-202$.

[6] S.H. Chen and C.H. Hsieh, Graded mean integration representation of generalized fuzzy number. J. Chin. Fuzzy Syst. Assoc. 5 (1999) $1-7$

[7] C. D'Ambrosio, R. Cerulli and M. Gentili, The optimal value range problem for the interval (immune) transportation problem. Omega 95 (2020) 102059.

[8] P. Das, S.K. De and S.S. Sana, An EOQ model for time dependent backlogging over idle time: a step order fuzzy approach. Int. J. Appl. Comput. Math. 1 (2014) 1-17.

[9] S.K. De and I. Beg, Triangular dense fuzzy sets and new defuzzification methods. J. Intell. Fuzzy Syst. 31 (2016) $469-477$.

[10] S.K. De and I. Beg, Triangular dense fuzzy Neutrosophic sets. Neutrosophic Sets Syst. 13 (2016) 1-12.

[11] S.K. De and G.C. Mahata, Decision of a fuzzy inventory with fuzzy backorder model under cloudy fuzzy demand rate. Int. J. Appl. Comput. Math. 3 (2017) 2593-2609. 
[12] S.K. De and G.C. Mahata, A production-inventory model with imperfect production process and partial backlogging under learning considerations in fuzzy random environments. J. Intell. Manuf. 28 (2017) 883-897.

[13] S.K. De and G.C. Mahata, A comprehensive study of an economic order quantity model under fuzzy monsoon demand. Sadhana 44 (2019) 89-101.

[14] S.K. De and G.C. Mahata, A cloudy fuzzy economic order quantity model for imperfect-quality items with allowable proportionate discounts. Int. J. Ind. Eng. 15 (2019) 571-583.

[15] S.K. De and G.C. Mahata, An EPQ model for three-layer supply chain with partial backordering and disruption: triangular dense fuzzy lock set approach. Sadhana 44 (2019) 177-192.

[16] S.K. De and G.C. Mahata, A production inventory supply chain model with partial backordering and disruption under triangular linguistic dense fuzzy lock set approach. Soft Comput. 24 (2020) 5053-5069.

[17] S.K. De and S.S. Sana, Fuzzy order quantity inventory model with fuzzy shortage quantity and fuzzy promotional index. 31 Econ. Model. (2013) 351-358.

[18] S.K. De and S.S. Sana, Backlogging EOQ model for promotional effort and selling price sensitive demand - an intuitionistic fuzzy approach. Ann. Oper. Res. 233 (2015) 57-76.

[19] S.K. De and S.S. Sana, The $(p, q, r, l)$ model for stochastic demand under Intuitionistic fuzzy aggregation with Bonferroni mean. J. Intell. Manuf. 29 (2018) 1753-1771.

[20] S.K. De, P.K. Kundu and A. Goswami, An economic production quantity inventory model involving fuzzy demand rate and fuzzy deterioration rate. J. Appl. Math. Comput. 12 (2003) 251-260.

[21] H. Deng, Comparing and ranking fuzzy numbers using ideal solutions. Appl. Math. Model. 38 (2014) $1638-1646$.

[22] R. Ezzati, T. Allahviranloo, S. Khezerloo and M. Khezerloo, An approach for ranking of fuzzy numbers. Expert Syst. App. 39 (2012) 690-695.

[23] T. Hajjari and S. Abbasbandy, A note on "The revised method of ranking LR fuzzy number based on deviation degree". Expert Syst. App. 39 (2011) 13491-13492.

[24] P.A. Hayek and M.K. Salameh, Production lot sizing with the reworking of imperfect quality items produced. Prod. Plan. Control 12 (2001) 584-590.

[25] M. Karimi-Nasab and K. Sabri-Laghaie, Developing approximate algorithms for EPQ problem with process compressibility and random error in production/inspection. Int. J. Prod. Res. 52 (2014) 2388-2421.

[26] N. Kazemi, E. Shekarian, L.E. Cárdenas-Barrón and E.U. Olugu, Incorporating human learning into a fuzzy EOQ inventory model with backorders. Comput. Ind. Eng. 87 (2015) 540-542.

[27] N. Kazemi, E.U. Olugu, A.R. Salwa Hanim and R.A.B.R. Ghazilla, Development of a fuzzy economic order quantity model for imperfect quality items using the learning effect on fuzzy parameters. J. Intell. Fuzzy Syst. 28 (2015) $2377-2389$.

[28] N. Kazemi, E.U. Olugu, A.R. Salwa Hanim and R.A.B.R. Ghazilla, A fuzzy EOQ model with backorders and forgetting effect on fuzzy parameters: an empirical study. Comput. Ind. Eng. 96 (2016) 140-148.

[29] A. Kumar, P. Singh, P. Kaur and A. Kaur, A new approach for ranking of L-R type generalized fuzzy numbers. Expert Syst. App. 38 (2011) 10906-10910.

[30] B. Maddah, M.K. Salameh and L. Moussawi-Haidar, Order overlapping: a practical approach for preventing shortages during screening. Comput. Ind. Eng. 58 (2010) 691-695.

[31] G.C. Mahata, A production-inventory model with imperfect production process and partial backlogging under learning considerations in fuzzy random environments. J. Intel. Manuf. 28 (2017) 883-897.

[32] G.C. Mahata and A. Goswami, An EOQ model for deteriorating items under trade credit financing in the fuzzy sense. Prod. Plan. Control 18 (2007) 681-692.

[33] G.C. Mahata and A. Goswami, Fuzzy inventory models for items with imperfect quality and shortage backordering under crisp and fuzzy decision variables. Comput. Ind. Eng. 64 (2013) 190-199.

[34] G.C. Mahata and P. Mahata, Analysis of a fuzzy economic order quantity model for deteriorating items under retailer partial trade credit financing in a supply chain. Math. Comput. Model. 53 (2011) 1621-1636.

[35] G.C. Mahata, A. Goswami and D.K. Gupta, A joint economic-lot-size model for purchaser and vendor in fuzzy sense. Comput. Math. App. 50 (2005) 1767-1790.

[36] L. Moussawi-Haidar, M. Salameh and W. Nasr, An instantaneous replenishment model under the effect of a sampling policy for defective items. Appl. Math. Modell. 37 (2013) 719-727.

[37] L. Moussawi-Haidar, M. Salameh and W. Nasr, Effect of deterioration on the instantaneous replenishment model with imperfect quality items. Appl. Math. Modell. 38 (2014) 5956-5966.

[38] S. Papachristos and I. Konstantaras, Economic ordering quantity models for items with imperfect quality. Int. J. Prod. Econ. 100 (2006) 148-154.

[39] K.S. Park, Fuzzy-set theoretic interpretation of economic order quantity. IEEE Transactions on Systems, Man, and Cybernetics SMC-17 (1987) 1082-1084.

[40] E.L. Porteus, Optimal lot sizing, process quality improvement and setup cost reduction. Oper. Res. 34 (1986) $137-144$.

[41] M.J. Rosenblatt and H.L. Lee, Economic production cycles with imperfect production processes. IIE Trans. 18 (1986) $48-55$.

[42] S.M. Ross, Introduction to Probability Models. Academic Press, New York (1993).

[43] M.K. Salameh and M.Y. Jaber, Economic production quantity model for items with imperfect quality. Int. J. Prod. Econ. 64 (2000) 59-64.

[44] R.L. Schwaller, EOQ under inspection costs. Prod. Inventory Manage. J. 29 (1988) 22-24. 
[45] W. Shih, Optimal inventory policies when stockouts result from defective products. Int. J. Prod. Res. 18 (1980) 677-685.

[46] E.A. Silver, Establishing the reorder quantity when amount received is uncertain. INFOR 14 (1976) 32-39.

[47] G. Sommer, Fuzzy inventory scheduling. In: Applied Systems and Cybernetics. Pergamon Press, New York (1981) 3052-3060.

[48] M.I.M. Wahab and M.Y. Jaber, Economic order quantity model for items with imperfect quality, different holding costs, and learning effects: a note. Comput. Ind. Eng. 58 (2010) 186-190.

[49] Z.X. Wang, Y.J. Liu, Z.P. Fan and B. Feng, Ranking L-R fuzzy number based on deviation degree. Inf. Sci. 179 (2009) 2070-2077.

[50] I.P. Wright, Factors affecting the cost of airplanes. J. Aeronautic Sci. 3 (1936) 122-128.

[51] P. Xu, X. Su, J. Wu, X. Sun, Y. Zhang and Y. Deng, A note on ranking generalized fuzzy numbers. Expert Syst. App. 39 (2012) 6454-6457.

[52] R.R. Yager, A procedure for ordering fuzzy subsets of the unit interval. Inf. Sci. 24 (1981) 143-161.

[53] H.F. Yu, W.K. Hsu and W.J. Chang, EOQ model where a portion of the defectives can be used as perfect quality. Int. J. Syst. Sci. 43 (2012) 1689-1698.

[54] V.F. Yu, H.T.X. Chi, L.Q. Dat, P.N.K. Phuc and C.W. Shen, Ranking generalized fuzzy numbers in fuzzy decision making based on the left and right transfer coefficients and areas. Appl. Math. Model. 37 (2013) 8106-8117.

[55] L.A. Zadeh, Fuzzy sets. Inf. Control 8 (1965) 338-356.

[56] X.I.N. Zhang and Y. Gerchak, Joint lot sizing and inspection policy in an EOQ model with random yield. IIE Trans. 22 (1990) 41-47.

[57] F. Zhang, J. Ignatius, C.P. Lim and Y. Zhao, A new method for ranking fuzzy numbers and its application to group decision making. Appl. Math. Modell. 38 (2014) 1563-1582. 